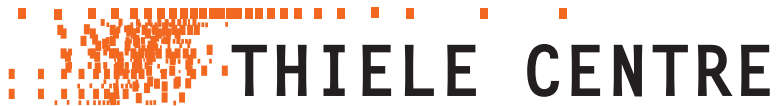 \\ FOR APPLIED MATHEMATICS IN NATURAL SCIENCE
}

Stability results for convex bodies

in geometric tomography

\section{Markus Kiderlen}




\section{Stability results for convex bodies in geometric tomography}

This Thiele Research Report is also Research Report number 491 in the Stochastics Series at Department of Mathematical Sciences, University of Aarhus, Denmark. 



\title{
STABILITY RESULTS FOR CONVEX BODIES IN GEOMETRIC TOMOGRAPHY
}

\author{
MARKUS KIDERLEN
}

\begin{abstract}
We consider the question in how far a convex body (non-empty compact convex set) $K$ in $n$-dimensional space is determined by tomographic measurements (in a broad sense). Usually these measurements are derived from $K$ by geometrical operations like sections, projections and certain averages of those. We restrict to tomographic measurements $F(K, \cdot)$ that can be written as function on the unit sphere and depend additively on an analytical representation $Q(K, \cdot)$ of $K$. The first main result states that $F(K, \cdot)$ is a multiplier-rotation operator of $Q(K, \cdot)$ whenever the tomographic data depends continuously and rotationally covariant on $K$. For $n \geq 3$, these operators are classical multiplier transforms.

We then turn to stability results stating that two convex bodies whose tomographic measurements are close to one another must be close in an appropriate metric on the family of convex bodies. We improve the Hölder exponents of known stability results for these transforms. The key idea for this improvement is to use the fact that support functions of convex bodies are elements of any spherical Sobolev space of derivative order less than $3 / 2$. As the analytical representation $Q(K, \cdot)$ may be a power of the support function, a power of the radial function, or a surface area measure, the class of tomographic data considered here is quite large. This is illustrated by many examples ranging from classical projection and section functions to directed tomographic transforms.
\end{abstract}

\section{INTRODUCTION AND MAIN RESULTS}

A classical result of ALEKSANDROV [1] states that an origin-symmetric convex body (non-empty compact convex set) $K$ in $\mathbb{R}^{n}$ with interior points is uniquely determined by its shadow areas on all hyperplanes. More precisely, let $u^{\perp}$ be the linear hyperplane with unit normal vector $u$ in the unit sphere $S^{n-1}$ and let $V_{n-1}\left(K \mid u^{\perp}\right)$ be the shadow area of $K$ in direction $u$, that is the $(n-1)$-dimensional volume of the orthogonal projection $K \mid u^{\perp}$ of $K$ on $u^{\perp}$. Aleksandrov used the fact that the shadow area in direction $u$ can be written as

$$
V_{n-1}\left(K \mid u^{\perp}\right)=\frac{1}{2} \int_{S^{n-1}}|\langle u, v\rangle| S_{n-1}(K, d v)
$$

where $\langle u, v\rangle$ is the usual inner product of $u$ and $v$ and $S_{n-1}(K, \cdot)$ is the surface area measure (of order $n-1$ ) of $K$, cf. [32]. As the even part of the latter determines a full-dimensional origin-symmetric convex body uniquely, a uniqueness proof is reduced to showing that the cosine transform $\mathrm{C}: \mu \mapsto \int_{S^{n-1}}|\langle\cdot, v\rangle| \mu(d v)$ determines the even part of a measure $\mu$. One way to do so is to use spherical harmonic analysis and to show that the cosine transform is a multiplier transformation: there is a sequence of real numbers $a_{0}, a_{1}, \ldots$ such that if $\mu \sim \sum_{k=0}^{\infty} \mu_{k}$ denotes the (formal and condensed)

Key words and phrases. Convex body, support function, radial function, surface area measure, geometric tomography, multiplier transform, multiplier-rotation transform, stability estimate, spherical Sobolev space.

Supported by the Carlsberg foundation. 
spherical harmonic expansion of $\mu$, then $\sum_{k=0}^{\infty} a_{k} \mu_{k}$ is the spherical harmonic expansion of its cosine transform. Explicit calculation shows that $a_{2 k}$ is nonzero for all $k$ implying that the even part of $\mu$ is indeed determined by $\mathrm{C}(\mu)$, and Aleksandrov's uniqueness result holds.

This method of proof is known to extend to other tomographic measurements, and we will give many examples below. They all have in common, that the tomographic data in question can be written as a multiplier transformation of some analytical representation of $K$. The first theorem presents a common framework for results of this type. To specify the way in which the tomographic measurements depend on an analytical representation of $K$, we return to the motivating example of shadow areas. Due to (1) and the fact that the Blaschke sum $K \# K^{\prime}$ of two convex bodies $K$ and $K^{\prime}$ with interior points is defined by means of the addition of their surface area measures, the tomographic data

$$
F(K, u)=V_{n-1}\left(K \mid u^{\perp}\right), \quad u \in S^{n-1},
$$

depends Blaschke additively on the convex body $K$, i.e.

$$
F\left(K \# K^{\prime}, \cdot\right)=F(K, \cdot)+F\left(K^{\prime}, \cdot\right) .
$$

Generalizing this property, we will work with tomographic data $F(K, \cdot)$ that satisfies

$$
F(K \diamond M, \cdot)=F(K, \cdot)+F(M, \cdot),
$$

where $\diamond$ may stand for Blaschke addition [32, p. 394], Minkowski addition [32, p. 41], FIREY's $p$-addition [12] or the radial addition [21, p. 19] of convex bodies. By definition of the Blaschke sum, (2) implies

$$
\begin{aligned}
S_{n-1}(K, \cdot)+S_{n-1}\left(K^{\prime}, \cdot\right) & =S_{n-1}(M, \cdot)+S_{n-1}\left(M^{\prime}, \cdot\right) \\
\Rightarrow \quad F(K, \cdot)+F\left(K^{\prime}, \cdot\right) & =F(M, \cdot)+F\left(M^{\prime}, \cdot\right)
\end{aligned}
$$

for all convex bodies $K, K^{\prime}, M, M^{\prime}$ with interior points. Hence, $F$ depends additively on the $(n-1)$-st surface area measure. More generally, we say that $F$ depends additively on an analytical representation $Q(K, \cdot)$ of $K$, if (4) holds with $Q(K, \cdot)$ replacing $S_{n-1}(K, \cdot)$. Here, $Q(K, \cdot)$ can either be a power of the support function, a power of the radial function or a $j$-th order surface area measure of $K, j=1, \ldots, n-1$. We note that the expression analytical is used here as opposed to geometric and does not involve any differentiability assumptions. It is convenient to replace (3) by the additive dependence condition on $Q(K, \cdot)$ (in the sense of $(4)$ ), as the latter does not require that the family $\{Q(K, \cdot): K$ is a convex body $\}$ is closed under addition. For example, if $F(K, \cdot)$ is the $j$-th order girth function with $j \in\{2, \ldots, n-2\}$ then $F$ depends additively on the surface area measure $S_{j}(K, \cdot)$ of order $j$, but condition (3) cannot be written down, as the addition of two $j$-th order surface area measures is not necessarily a $j$-th order surface area measure itself.

Besides the additivity assumption, we impose that the tomographic data is a square integrable function on the unit sphere, depends continuously on the underlying convex body and is independent of rotations of the coordinate system. We summarize these conditions using $\mathcal{K}$ as notation for the family of all convex bodies in $\mathbb{R}^{n}$, endowed with the Hausdorff metric. For this and other concepts in convex geometry, SCHNEIDER's book [32] is a standard reference.

(i) For all $K \in \mathcal{K}, F(K, \cdot)$ is an element of $L^{2}$, the Hilbert space of square integrable functions on the unit sphere,

(ii) $K \mapsto F(K, \cdot)$ is continuous, 
(iii) $F(K, \cdot)$ intertwines the action of the special rotation group $S O_{n}$, meaning that

$$
\vartheta F(K, \cdot):=F\left(K, \vartheta^{-1}(\cdot)\right)=F(\vartheta K, \cdot), \quad \vartheta \in S O_{n},
$$

holds for all $K \in \mathcal{K}$,

(iv) there is an analytical representation $Q(K, \cdot)$ of $K$ such that $F(K, \cdot)$ depends additively on $Q(K, \cdot)$, i.e. for all $K, K^{\prime}, M, M^{\prime} \in \mathcal{K}$,

$$
F(K, \cdot)+F(M, \cdot)=F\left(K^{\prime}, \cdot\right)+F\left(M^{\prime}, \cdot\right)
$$

holds whenever

$$
Q(K, \cdot)+Q(M, \cdot)=Q\left(K^{\prime}, \cdot\right)+Q\left(M^{\prime}, \cdot\right) .
$$

An analytical representation $Q(K, \cdot)$ of $K$ can either be a power of its support function $h_{K}^{\theta}, \theta \neq 0$, a power of its radial function $\rho_{K}^{\theta}, \theta \neq 0$, or a surface area measure $S_{j}(K, \cdot), j \in\{1, \ldots, n-1\}$. In the cases $Q(K, \cdot)=h_{K}^{\theta}$, where $\theta$ is not an odd integer, and $Q(K, \cdot)=\rho_{K}^{\theta}$, we assume that $K$ contains the origin $o$. When $\theta$ is an even integer, $h_{K}^{\theta}$ does not determine $K$ uniquely (consider singletons), and this is why we impose $o \in K$ also in this case.

Under these assumptions, $F(K, \cdot)$ is essentially a multiplier transformation of $Q(K, \cdot)$.

Theorem 1. Let $F(K, \cdot)$ be a mapping satisfying $(i)$ - (iv) with analytical representation $Q(K, \cdot) \sim \sum_{k=0}^{\infty} Q_{k}(K, \cdot)$. Then there is a real sequence $\mathbf{a}=\left(a_{k}\right)$ and a sequence $\left(\vartheta_{k}\right)$ in $S O_{n}$ with

$$
F(K, \cdot) \sim \sum_{k=0}^{\infty} a_{k} \vartheta_{k} Q_{k}(K, \cdot)
$$

for all $K \in \mathcal{K}$. For $n \geq 3$ all the rotations $\vartheta_{k}$ are the identity. The sequence $\left(\left|a_{k}\right|\right)$ grows at most polynomially in $k$.

The numbers $a_{0}, a_{1}, a_{2}, \ldots$ are called multipliers of $F(K, \cdot)$. We refer to $F(K, \cdot)$ satisfying (5) as a multiplier-rotation operator. The two-dimensional case $(n=2)$ is qualitatively different, as the rotation group is Abelian. A multiplier-rotation operator can be written as a multiplier operator

$$
F(K, \cdot) \sim \sum_{k=0}^{\infty} a_{k} Q_{k}(K, \cdot)
$$

if and only if $K \mapsto F(K, \cdot)$ intertwines the action of the (general) rotation group $O_{n}$. Theorem 1 implies in particular that this is the case for all tomographic transforms if $n \geq 3$.

Note that the assumptions on $F(K, \cdot)$ are quite weak. In particular, it is assumed that $F(K, \cdot)$ depends continuously on $K$, not on $Q(K, \cdot)$. To show the multiplier property we therefore first prove that $K \mapsto F(K, \cdot)$ gives rise to an associated continuous linear transform $T$ on a sufficiently large class of spherical functions, and apply results of spherical harmonic analysis to T. SCHnEIDER [31], the author [27] and SCHUster [35] exploited this idea in the special cases where $Q(K, \cdot)$ is either the support function or the $(n-1)$-st surface area measure of $K$. We extend these arguments to general $Q(K, \cdot)$. Let $\mathcal{C}^{m}$ be the class of all $m$-times continuously differentiable functions, $m \in \mathbb{N}_{0}$. It is shown in Proposition 9 that the linear space spanned by $\{Q(K, \cdot): K \in \mathcal{K}\}$ contains $\mathcal{C}^{2}$, if $Q(K, \cdot)$ is a (non-vanishing) power of the support function or the radial function. In the case where $Q(K, \cdot)$ is a surface area measure, Proposition 10 shows that the family of all functions in $\mathcal{C}^{2\lceil(n+1) / 4\rceil}$ with centroid at the origin is contained in the linear 
span of $\{Q(K, \cdot): K \in \mathcal{K}\}$. (An integrable function $f$ is here identified with the signed measure $\mu$ by $d \mu(u)=f(u) d u)$.) These results, also of interest in their own right, allow to define $T$ on $\mathcal{C}^{m}$ for sufficiently large $m$.

Like in the special case of shadow areas, injectivity properties of $K \mapsto F(K, \cdot)$ depend on the injectivity properties of $Q(K, \cdot)$ and on the sequence of multipliers a. Define the sets

and

$$
\mathcal{K}_{\mathbf{a}, h^{\theta}}:= \begin{cases}\left\{K \in \mathcal{K}: h_{K}^{\theta} \sim \sum_{a_{k} \neq 0}\left(h_{K}^{\theta}\right)_{k}\right\}, & \theta \in 2 \mathbb{N}_{0}+1, \\ \left\{K \in \mathcal{K}: h_{K}^{\theta} \sim \sum_{a_{k} \neq 0}\left(h_{K}^{\theta}\right)_{k}, o \in K\right\}, & \theta \notin 2 \mathbb{N}_{0}+1,\end{cases}
$$

$$
\mathcal{K}_{\mathbf{a}, \rho^{\theta}}=\left\{K \in \mathcal{K}: \rho_{K}^{\theta} \sim \sum_{a_{k} \neq 0}\left(\rho_{K}^{\theta}\right)_{k}, o \in K\right\} .
$$

As $K$ is determined by $S_{j}(K, \cdot)$ only up to translation and only if $\operatorname{dim} K>j$ (cf. [32, Corollary 7.2.5]) we set

$$
\mathcal{K}_{\mathbf{a}, S_{j}}=\left\{K \in \mathcal{K}: S_{j}(K, \cdot) \sim \sum_{a_{k} \neq 0}\left(S_{j}(K, \cdot)\right)_{k}, \operatorname{dim} K>j, \text { and } s(K)=o\right\},
$$

where $s(K)$ is the Steiner point of $K$. If $F(K, \cdot)$ is satisfying (i) - (iv) and has a as multiplier sequence, then $K \mapsto F(K, \cdot)$ is injective on $\mathcal{K}_{\mathbf{a}, Q}$. To give some examples, assume that exactly the even multipliers $a_{2 k}, k=0,1,2, \ldots$, of $F$ are non-zero. Then $\mathcal{K}_{\mathbf{a}, h^{\theta}}=\mathcal{K}_{\mathbf{a}, \rho^{\theta}}$ is the family of origin-symmetric convex bodies and $\mathcal{K}_{\mathbf{a}, S_{j}}$ is the subfamily of origin-symmetric convex bodies of dimension at larger than $j$. Another typical example occurs, when exactly the odd multipliers and $a_{0}$ are non-zero. Then, $\mathcal{K}_{\mathbf{a}, h}$ is the family of bodies of constant width and $\mathcal{K}_{\mathbf{a}, S_{n-1}}$ is the family of full-dimensional centered bodies of constant brightness.

For later use we define for nonnegative $r$ and $R$ the families

$$
\mathcal{K}(R):=\left\{K \in \mathcal{K}: K \subset R B^{n}\right\} \quad \text { and } \quad \mathcal{K}(r, R):=\left\{K \in \mathcal{K}: r B^{n} \subset K \subset R B^{n}\right\},
$$

(where $B^{n}$ is the Euclidean unit ball in $\mathbb{R}^{n}$ ) and

$$
\mathcal{K}_{\mathbf{a}, Q}(R):=\mathcal{K}_{\mathbf{a}, Q} \cap \mathcal{K}(R), \quad \mathcal{K}_{\mathbf{a}, Q}(r, R):=\mathcal{K}_{\mathbf{a}, Q} \cap \mathcal{K}(r, R) .
$$

We return to the example of shadow areas from the introduction. Given uniqueness, it is natural to ask whether small deviations of corresponding shadow areas of two suitable convex bodies imply that the bodies are close to one another. Such a stability result has been shown by BOURGAIN AND Lindenstrauss [5]. We state it in the form given by Groemer [21]. For any $\gamma, r, R>0$, there is a constant $c_{1}=c_{1}(n, \gamma, r, R)>0$ such that

$$
\delta\left(K, K^{\prime}\right) \leq c_{1}\left\|V_{n-1}\left(K \mid u^{\perp}\right)-V_{n-1}\left(K^{\prime} \mid u^{\perp}\right)\right\|^{2 /(n(n+4))-\gamma}
$$

holds for all origin-symmetric convex bodies $K, K^{\prime} \in \mathcal{K}(r, R)$. Here, $\delta\left(K, K^{\prime}\right)$ is the Hausdorff distance between $K$ and $K^{\prime}$ and $\|\cdot\|$ denotes the $L^{2}$-metric on the unit sphere (with respect to $u$ ). Independently of Bourgain And Lindenstrauss, Campi [8] has shown a similar stability result in the special case $n=3$ with an exponent $1 / 9-\gamma$, which is better than $2 / 21-\gamma$ in (6). This improvement is due to the fact that in [5], properties of first order derivatives of support functions are used, whereas CAMPI works with derivatives of these functions of (fractional) order $\eta<3 / 2$, close to $3 / 2$. The original motivation for the present work was the question whether CAMPI's improved stability result can be extended to higher dimensions. Firstly such an extension would require that any family of uniformly bounded support functions on $S^{n-1}$ is bounded with respect to the spherical Sobolev norm of derivative order $\eta$. This is proved and 
used in [8] for $n=3$; from another paper [9] of CAMPI, it easily follows that this is also true in arbitrary dimensions $n \geq 3$; see Proposition 7 below. The second main ingredient for CAMPI's approach is a bound depending on the dimension of the space of spherical harmonics of degree $k$. This dimension grows moderately (linear) in $k$ in $\mathbb{R}^{3}$, but at least quadratic for $n \geq 4$, which makes CAMPI's method unfit in higher dimensions. A closer look, however, reveals that a combination of CAMPI's ideas and the Poisson integral estimates exploited by Bourgain And Lindenstrauss leads to an improved exponent in (6) for arbitrary $n \geq 2$ : a special case of Theorem 4 states that $2 /(n(n+4))$ can be replaced by $2 /(n(n+1))$, which yields $1 / 6$ in $\mathbb{R}^{3}$.

We will now formulate stability results for general tomographic data satisfying conditions (i) - (iv). It turns out that these stability results only depend on the underlying analytical representation $Q(K, \cdot)$ and on the multipliers $a_{0}, a_{1}, a_{2}, \ldots$ of $F$. In all stability statements, we will therefore assume (5) instead of (i) - (iv), keeping in mind that this assumption is weaker. Considering the inverse problem to reconstruct $K$ from its tomographic data $F(K, \cdot) \sim \sum_{k=0}^{\infty} F_{k}$, relation (5) suggests, roughly speaking, to reconstruct $Q(K, \cdot)$ using $\sum_{k=0}^{\infty} a_{k}^{-1} \vartheta_{k}^{-1} F_{k}$ in a first step, and then reconstructing $K$ from this approximation of $Q(K, \cdot)$. The continuity properties of such an inversion therefore depend on the asymptotic behavior of $\left(\left|a_{k}\right|^{-1}\right)$. We will require that this behavior is polynomial: assume that there are non-negative constants $b$ and $\beta$ such that

$$
\left|a_{k}\right|^{-1} \leq b(1 \vee k)^{\beta}, \quad \text { for all } k \text { with } a_{k} \neq 0 .
$$

Here $a \vee b$ is the maximum of $a, b \in \mathbb{R}$. Before stating stability results, some examples are discussed, where the multipliers or at least their asymptotic behavior can be determined. These are examples, where $F(K, \cdot)$ can be written as an integral transform of $Q(K, \cdot)$. For instance, we have already seen that the shadow area function can be written as the cosine transform of the $(d-1)$ st surface area measure of $K$. We list the most common integral transforms and their properties. Their connection to tomographic measurements will be clarified later. All these transforms are defined here on the space $\mathcal{C}$ of continuous functions on $S^{n-1}$, but can be extended to weakly continuous transforms on the space of finite signed measures.

(a) The cosine transform, given by

$$
\mathrm{C}(f):=\int_{S^{n-1}}|\langle v, \cdot\rangle| f(v) d v, \quad f \in \mathcal{C},
$$

has the property that all its odd multipliers $a_{2 k+1}$ vanish. The even multipliers satisfy (7) with $\beta=(n+2) / 2$, and this exponent is best possible.

(b) The spherical Radon transform $\mathrm{R}(f)$ is defined by

$$
(\mathrm{R} f)(u):=\int_{S^{n-1} \cap u^{\perp}} f(v) d v, \quad f \in \mathcal{C},
$$

where integration is understood with respect to spherical Lebesgue measure on the great sphere orthogonal to $u \in S^{n-1}$. All the odd multipliers $a_{2 k+1}$ vanish; the even multipliers satisfy $(7)$ with $\beta=(n-2) / 2$, and this exponent is best possible.

(c) The sine transform, given by

$$
\mathrm{S}(f):=\int_{S^{n-1}} \sqrt{1-\langle v, \cdot\rangle^{2}} f(v) d v, \quad f \in \mathcal{C},
$$


coincides up to a multiplicative constant with the composition RC, (see e.g. [37, equation (5.4)]) and thus, again, all the odd multipliers $a_{2 k+1}$ vanish and the even multipliers satisfy (7) with $\beta=n$.

(d) The spherical cap transform. For $u \in S^{n-1}$ and fixed $\varphi \in(0, \pi)$ let

$$
C_{\varphi}(u):=\left\{v \in S^{n-1}:\langle u, v\rangle \geq \cos \varphi\right\}
$$

be the cap of all points on the unit sphere whose angle with $u$ is at most $\varphi$. The spherical cap transform or generalized Funk transform (with parameter $\varphi$ ) is given by

$$
\left(\mathrm{F}_{\varphi} f\right)(u):=\int_{C_{\varphi}(u)} f(v) d v, \quad f \in \mathcal{C}, u \in S^{n-1} .
$$

This transform represents a special case of generalized Minkowski-Funk transforms. These were treated by RUBIN [29] who determined the asymptotic behavior of the multipliers in the case where $\varphi$ is a rational multiple of $\pi$. Put

$$
r_{0}=\frac{n-2}{4}\left(1-2 \frac{\varphi}{\pi}\right)
$$

and assume that $\varphi / \pi=p / q \neq 1 / 2$ where the natural numbers $p$ and $q$ are relatively prime. [29, Theorem 3.2] implies that the sequence of multipliers a satisfies (7) with $\beta=n / 2$ if $q r_{0} \notin \mathbb{Z}$. In the case $q r_{0} \in \mathbb{Z}$, the value $\beta=(n+2) / 2$ is best possible. Note, however, that this does not imply injectivity of $F_{\varphi}$, as $(7)$ involves only the nonvanishing multipliers. By the Funk-Hecke formula, the multiplier $a_{k}, k>1$, coincides essentially with the value of the Legendre polynomial $P_{k-1}^{d+2}(x)$ at $x=\cos \varphi$; see [29]. Using this, an injectivity proof would require to determine if a given value $\cos \varphi$ is the zero of at least one of the polynomials in the infinite sequence $\left(P_{k}^{d+2}(x)\right)_{k}$. RUBIN determined a strong bound $\bar{k}=\bar{k}(n, \varphi) \in \mathbb{Z}$ such that $a_{k}=0$ implies $2 \leq k \leq \bar{k}$. If $\bar{k}<2$ (or none of the finitely many polynomials $P_{1}^{d+2}(x), \ldots, P_{\bar{k}-1}^{d+2}(x)$ vanishes at $x=\cos \varphi)$ then $\mathrm{F}_{\varphi}$ is injective. As examples, he mentions that $\mathrm{F}_{\varphi}$ is injective on $\mathbb{R}^{3}$ for $\varphi=\pi / 3$ and $\varphi=\pi / 4$. The cases where $\varphi$ is not a rational multiple of $\pi$ are much more involved, but it is known that (7) cannot hold with $\beta<(n+2) / 2$.

The special case $\varphi=\pi / 2$, excluded in the above discussion, is easier to handle. As integration is extended over closed half spheres, the corresponding transform is called hemispherical transform $\mathrm{H}=\mathrm{F}_{\pi / 2}$. All the even multipliers $a_{2 k}$ of $\mathrm{H}$ vanish with the exception of $a_{0}$. The odd multipliers satisfy (7) with with $\beta=n / 2$ being the best possible exponent.

More details on the first two examples and on the hemispherical transform can be found e.g. in Groemer's book [21]. StEPAnov [39] discusses general spherical integral operators of convolution type with an integrable kernel, a setting which does not include the spherical Radon transform. According to the underlying analytical representation, stability results are given in Subsections 1.1 (power of the support function) and 1.2 (power of the radial function). In Subsection 1.3 we treat tomographic data which depends additively on the $j$-th surface area measure. Applications of the general results will also be given in these subsections. Most of them are well-known (see the detailed historical remarks in [21]), but we can in almost all cases provide better Hölder exponents.

1.1. Tomographic data from a power of the support function. In the case, where the tomographic data depends additively on a power of the support function, a 
stability result follows essentially from Hölder's inequality, in the form of Proposition 13. It is natural to formulate stability results in terms of the $L^{2}$-metric on $\mathcal{K}$, given by

$$
\delta_{2}\left(K, K^{\prime}\right):=\left\|h_{K}-h_{K^{\prime}}\right\|, \quad K, K^{\prime} \in \mathcal{K} .
$$

Using the known relation (23) below, stability results can be rephrased with the usual Hausdorff metric at the cost of a weaker exponent.

Theorem 2. Let $n \geq 2$. Assume that $F(K, \cdot)$ satisfies (5) with $Q(K, \cdot)=h_{K}^{\theta}, \theta \neq 0$, and that its multipliers satisfy (7). Then, for any $\gamma>0$ and $0<r \leq R$, there is a constant $c_{2}=c_{2}(n, \theta, b, \beta, \gamma, r, R)$ such that

$$
\delta_{2}\left(K, K^{\prime}\right) \leq c_{2}\left\|F(K, \cdot)-F\left(K^{\prime}, \cdot\right)\right\|^{\frac{3}{3+2 \beta}-\gamma}
$$

holds for all $K, K^{\prime} \in \mathcal{K}_{\mathbf{a}, h^{\theta}}(r, R)$.

If $\theta \geq 2$ or $\theta \in \mathbb{N}$, the set $\mathcal{K}_{\mathbf{a}, h^{\theta}}(r, R)$ can be replaced by $\mathcal{K}_{\mathbf{a}, h^{\theta}}(0, R)$ or $\mathcal{K}_{\mathbf{a}, h^{\theta}}(R)$, respectively.

As the exponent in (8) is not known to be optimal, it is futile to ask for an optimal leading constant. In the literature, special cases of Theorem 2 where treated, all of them with $\theta=1$. CAMPI [7] was apparently the first who stated a stability result for tomographic data from support functions: he established stability from the mean width of hyperplane shadows (see Application (a) below) in the case $n=3$. The corresponding integral transform is the spherical Radon transform. His article is based on earlier work of his own and uses Jensen's inequality (which, however, is applied in such a way that Hölder's inequality could be used instead); cf. [6]. Goodey And Groemer [18] make explicit use of Hölder's inequality to extend CAMPI's result to higher dimensions. Both papers use derivatives of support functions up to order 1 instead of $3 / 2$, and therefore obtain the weaker Hölder exponent $1 /(1+\beta)$. This weaker exponent is also the one established in [26, Proposition 4], where slightly stronger assumptions than in Theorem 2 were used.

\section{Applications.}

(a) Support function power integrals of central hyperplane projections. For a convex body $K \subset \mathbb{R}^{n}$ (with $o \in K$ if $\theta \notin 2 \mathbb{N}_{0}+1$ ), we call

$$
J_{\theta}^{n}(K)=\int_{S^{n-1}} h_{K}^{\theta}(u) d u
$$

the support function power integral of dimension $n$ and order $\theta$. The tomographic measurements, given by

$$
F(K, u)=J_{\theta}^{n-1}\left(K \mid u^{\perp}\right)=\left(\mathrm{R} h_{K}^{\theta}\right)(u), \quad u \in S^{n-1},
$$

can be written as spherical Radon transform of $h_{K}^{\theta}$. Hence, Theorem 2 can be applied with $\beta=(n-2) / 2$. $\mathcal{K}_{\mathbf{a}, h^{\theta}}$ now is the family of origin-symmetric convex bodies. It follows that for any $\gamma>0$ and $0<r \leq R$, there is a constant $c_{3}=c_{3}(n, \theta, \gamma, r, R)$ such that

$$
\delta_{2}\left(K, K^{\prime}\right) \leq c_{3}\left\|J_{\theta}^{n-1}\left(K \mid(\cdot)^{\perp}\right)-J_{\theta}^{n-1}\left(K \mid(\cdot)^{\perp}\right)\right\|^{3 /(n+1)-\gamma}
$$

holds for all $K, K^{\prime} \in \mathcal{K}_{\mathbf{a}, h^{\theta}}(r, R)$ (or $K, K^{\prime} \in \mathcal{K}_{\mathbf{a}, h^{\theta}}(R)$, when $\left.\theta \in \mathbb{N}\right)$. The mean width

$$
\bar{w}(K)=\frac{2}{\varpi_{n}} \int_{S^{n-1}} h_{K}(u) d u=\frac{2}{\varpi_{n}} J_{1}^{n}(K)
$$


is essentially the support function power integral of order 1 (with $\varpi_{n}$ being the surface area of $S^{n-1}$ ). Hence

$$
\delta_{2}\left(K, K^{\prime}\right) \leq \frac{\varpi_{n} c_{3}}{2}\left\|\bar{w}^{\prime}\left(K \mid(\cdot)^{\perp}\right)-\bar{w}^{\prime}\left(K \mid(\cdot)^{\perp}\right)\right\|^{3 /(n+1)-\gamma}
$$

for all origin-symmetric $K, K^{\prime} \subset R B^{n}$. The tomographic data $\bar{w}^{\prime}\left(K \mid u^{\perp}\right)$ is the mean width of $K \mid u^{\perp}$; the dash indicates that the mean width is taken relative to the hyperplane $u^{\perp}$. However, this is not essential, as $u \mapsto \bar{w}\left(K \mid u^{\perp}\right)$ (with $K \mid u^{\perp}$ considered as a lower dimensional subset of $\left.\mathbb{R}^{n}\right)$ is proportional to $u \mapsto \bar{w}^{\prime}\left(K \mid u^{\perp}\right)$. For $n \geq 3$, the Hölder exponent in (10) is better than 2/ $n$ obtained by GoodeY AND Groemer [18]. It should be remarked that their result does not require $K, K^{\prime} \subset R B^{n}$, as their leading constant depends on certain intrinsic volumes of $K$ and $K^{\prime}$.

Note that $J_{1}^{n}(K)$ also has an interpretation as the probability that an isotropically and uniformly chosen affine hyperplane hits $K$. Therefore, (10) can be interpreted as a stability result for the tomographic data obtained from hitting probabilities with UIR (uniform isotropic random) hyperplanes in $u^{\perp}$. Replacing UIR hyperplanes by onepoint sampled hyperplanes, a stability result can be derived from (9) with $\theta=n$. This is made precise in [28].

(b) Mean width and Steiner point from hyperplane projections. As the mean widths of hyperplane projections of two convex bodies only allow to compare the even parts of their support functions, SCHNEIDER [34] suggests to use additional information by comparing also the Steiner points of the two projections. The Steiner point of a projection satisfies

$$
s^{\prime}\left(K \mid u^{\perp}\right)=\frac{n-1}{\varpi_{n-1}} \int_{S^{n-1} \cap u^{\perp}} v h_{K}(v) d v=\frac{n-1}{\varpi_{n-1}}(\mathrm{R} f)(u)
$$

with $f(v)=v h_{K}(v)$ (and we apply the spherical Radon transform component-wise to the vector-valued function $f$ ). The even part of $f$, and thus the odd part of $h_{K}$ is determined by this information. Following the line of arguments given by Schneider, using the better estimate for the Hölder exponent, we obtain that

$$
\left\|\bar{w}^{\prime}\left(K \mid(\cdot)^{\perp}\right)-\bar{w}^{\prime}\left(K \mid(\cdot)^{\perp}\right)\right\| \leq \varepsilon
$$

and

implies

$$
\left\|\left|s^{\prime}\left(K \mid(\cdot)^{\perp}\right)-s^{\prime}\left(K \mid(\cdot)^{\perp}\right)\right|\right\| \leq \varepsilon
$$

$$
\delta_{2}\left(K, K^{\prime}\right) \leq c_{4} \varepsilon^{3 /(n+1)-\gamma}
$$

for all $K, K^{\prime} \in \mathcal{K}(R)$, where $c_{4}=c_{4}(n, \gamma, R)$.

(c) Mean directed projection function of order one and averages. Like in (b), the motivation to consider this tomographic data is that the mean widths of (hyper-)plane projections only determine the even part of the support function of the unknown convex body. GoODEY AND WEIL [15] suggest therefore to consider directed measurements of the projections. Let $\mathcal{L}_{k}^{n}$ be the Grassmannian of all $k$-dimensional linear subspaces of $\mathbb{R}^{n}$ and let $u^{+}$be the closed half space bounded by $u^{\perp}$ (containing $u$ ). In view of the definition of mean width, GoODEY AND WEIL introduce for $1 \leq k \leq n-1$ the function

$$
\bar{w}_{1}(K ; L, u):=\frac{2}{\varpi_{k}} \int_{S^{n-1} \cap L \cap u^{+}} h_{K}(u) d u
$$

of pairs $(L, u)$ with $L \in \mathcal{L}_{k}^{n}, u \in S^{n-1} \cap L$, which we call directed projection mean width. Integration is understood here with respect to spherical Lebesgue measure on 
$S^{n-1} \cap L$. (In [15] a different normalization is used and the above function is denoted by $p_{1, j}(K ; L, u)$. More importantly, such functions are discussed in a much more general framework where directed versions of all classical projection functions of lower order are stated using tensor formulas.) Applying Theorem 2 to the hemispherical transform in $L$ and the spherical Radon transform in suitable $(k+1)$-dimensional spaces, a stability result can be derived; see [23] for a proof in a similar situation. Let $K, K^{\prime} \in \mathcal{K}(R)$ be given and assume that there is an $\varepsilon>0$ such that, for all $L \in \mathcal{L}_{k}^{n}$ and all $u \in S^{n-1} \cap L$ we have

$$
\left|\bar{w}_{1}(K ; L, u)-\bar{w}_{1}\left(K^{\prime} ; L, u\right)\right| \leq \varepsilon .
$$

Then, for any $\gamma>0$ there is a constant $c_{5}=c_{5}(n, \gamma, R)$ such that

$$
\delta_{2}\left(K, K^{\prime}\right) \leq c_{5} \varepsilon^{3 /(k+2)-\gamma} .
$$

As $K$ appears to be overdetermined by $\bar{w}_{1}(K ; \cdot)$, GoODEY AND WeIL [15] suggest to consider averages of the directed projection mean width, where averaging is understood with respect to all $k$-dimensional half-spaces (containing $u$ ) in the half-space $u^{+}$:

$$
F(K, u):=\int_{\mathcal{L}_{k-1}^{u \perp}} \bar{w}_{1}\left(K ; L_{u}, u\right) d L, \quad u \in S^{n-1} .
$$

Here $\mathcal{L}_{k-1}^{u^{\perp}}$ is the Grassmannian of all $(k-1)$-dimensional subspaces in $u^{\perp}, L_{u}$ is the linear space spanned by $L$ and $u$, and integration is understood with respect to the usual invariant probability measure on $\mathcal{L}_{k-1}^{u^{\perp}}$. Clearly, $F(K, \cdot)$ depends additively on the analytical representation $h_{K}$ of $K$ and is therefore a multiplier transformation. It is noted in [15] that in the cases $k \leq(n+2) / 2$ and $k>(2 n+1) / 3$, for $n \neq 4$, as well as $k=2$ for $n=4$, all multipliers are non-zero and satisfy (7) with $\beta=n$; see also [14]. Theorem 2 implies for these pairs $(k, n)$ of parameters that for any positive $\gamma$ and $R$, there is a constant $c_{6}=c_{6}(n, \gamma, R)$ with

$$
\delta_{2}\left(K, K^{\prime}\right) \leq c_{6}\left\|F(K, \cdot)-F\left(K^{\prime}, \cdot\right)\right\|^{\frac{3}{2 n+3}-\gamma}
$$

holding for all $K, K^{\prime} \in \mathcal{K}(R)$. It should be noted that there are infinitely many pairs $(k, n)$ for which $K$ is not determined by $F(K, \cdot)$.

Definition (11) is not the only possible way to define a directed version of the (first order) projection function. As the mean width of $K$ coincides with $2 / \varpi_{n} S_{1}\left(K, S^{n-1}\right)$, another possible directed extension would be the function

$$
\bar{w}_{2}(K ; L, u):=\frac{2}{\varpi_{k}} S_{1}\left(K \mid L, u^{+} \cap L \cap S^{n-1}\right)-\frac{1}{\varpi_{k}} S_{1}\left(K \mid L, u^{\perp} \cap L \cap S^{n-1}\right) ;
$$

cf. the more general directed tomographic data defined by (20) in Subsection 1.3 below. The same averaging process as in (12) leads to the tomographic data

$$
F(K, u):=\int_{\mathcal{L}_{k-1}^{u \perp}} \bar{w}_{2}\left(K ; L_{u}, u\right) d L, \quad u \in S^{n-1} .
$$

GOODEY AND WeIL [16] denote this function (with a different normalization) by $\bar{v}_{1, k}(K ; \cdot)$ and show that it can be expressed as an average of lower dimensional hemispherical transforms of a differential operator, applied to $h_{K}$. This shows that $F(K, \cdot)$ depends additively on the support function. Furthermore $F: \mathcal{K} \rightarrow L^{2}$ depends continuously on $K$. This follows from the fact that if $K_{1}, K_{2}, \ldots$ is a sequence of convex bodies converging to some $K \in \mathcal{K}$, and $L \in \mathcal{L}_{k}^{n}$ is fixed then $\bar{w}_{2}\left(K_{m} ; L, u\right)$ converges to $\bar{w}_{2}(K ; L, u)$ for almost all $u$ in the unit sphere in $L$; see $[16$, p. 47]. Hence, the 
assumptions of Theorem 1 are satisfied and $F(K, \cdot)$ is a multiplier transformation of the support function of $K$. In [16], this was derived in a different manner and it was shown that for $2 \leq k<(2 n-3) / 5$ or $(n-2) / 2 \leq k \leq n-1$ all multipliers (with the exception of $a_{1}$ ) are non-zero and satisfy (7) with $\beta=n$.

Theorem 2 now implies that for these pairs $(k, n)$ of parameters and any positive $\gamma$ and $R$, there is a constant $c_{7}=c_{7}(n, \gamma, R)$ such that

$$
\delta_{2}\left(K, K^{\prime}\right) \leq c_{7}\left\|F(K, \cdot)-F\left(K^{\prime}, \cdot\right)\right\|^{\frac{3}{2 n+3}-\gamma}
$$

holds for all $K, K^{\prime} \in \mathcal{K}(R)$ with Steiner point at the origin. In view of (26), the Hölder exponent $2 /(n+1)^{2}$ for stability in the Hausdorff metric in [16, Theorem 7 ] can therefore be improved to $6 /((n+1)(2 n+3))-\gamma$. There are also know pairs $(k, n)$ of parameters for which $F(K, \cdot)$ does not determine $K$ uniquely, e.g. those in $\{(k, 5 k+4): k=1,2, \ldots\}$.

(d) Mean $k$-dimensional projection. Let $1 \leq k \leq n-1$ be given and set

$$
F(K, u):=\int_{\mathcal{L}_{k}^{n}} h_{K \mid L}(u) d L, \quad u \in S^{n-1},
$$

where integration is understood with respect to the invariant probability measure on $\mathcal{L}_{k}^{n}$ and the set $K \mid L$ is considered as a (lower dimensional) convex body in $\mathbb{R}^{n}$. Obviously, $F(K, \cdot)$ satisfies the conditions (i)-(iv). Considering (iv), $F(K, \cdot)$ is additive with respect to $Q(K, \cdot)=h_{K}$, as $h_{K \mid L}$ equals the restriction of $h_{K}$ to the unit sphere in $L$. It is shown in [26], that $F(K, \cdot)$ can be written as an integral transform of $h_{K}$ with a trigonometric integral kernel, but the multipliers and their asymptotic behavior are only known in special cases. In particular, there are pairs $(k, n)$ with $k>1$ such that $F(K, \cdot)$ does not determine the convex body $K \subset \mathbb{R}^{n}$ uniquely. One such pair is $(k, n)=(2,14)$; see Goodey [17]. We refer to [26] for a detailed discussion and reference list and only note two special cases with improved Hölder exponents: For projections on hyperplanes, SPRIESTERSBACH [38] (see the correcting comment in [26]) obtained a stability result with Hölder exponent $1 / 2$. This can be improved to

$$
\delta_{2}\left(K, K^{\prime}\right) \leq c_{8}\left\|F(K, \cdot)-F\left(K^{\prime}, \cdot\right)\right\|^{3 / 5-\gamma},
$$

holding for all convex bodies $K$ and $K^{\prime}$ in a given ball $R B^{n}, k=n-1$. For general $k \in\{2, \ldots, n-1\}$ with the property that $(k, n)$ is not an exceptional pair, one would expect

$$
\delta_{2}\left(K, K^{\prime}\right) \leq c_{9}\left\|F(K, \cdot)-F\left(K^{\prime}, \cdot\right)\right\|^{3 /(3+2(n-k))-\gamma}
$$

for all $K, K^{\prime} \in \mathcal{K}(R)$. Combining the refined estimates in the present work with the arguments leading to [26, Theorem 1], it follows that (15) actually holds for originsymmetric $K$ and $K^{\prime}$ if $k$ is even and for bodies $K$ and $K^{\prime}$ of constant width if $k$ is odd.

1.2. Tomographic data from a power of the radial function. Working with powers of radial functions, the radial $L^{2}$-distance

$$
\rho_{2}\left(K, K^{\prime}\right)=\left\|\rho_{K}-\rho_{K^{\prime}}\right\|
$$

will appear in stability estimates for convex bodies $K$ and $K^{\prime}$ with $o$ in their interior. As before, these results can be stated in terms of the Hausdorff distance using (26), below, at the cost of a weaker Hölder exponent. Note that the Hölder exponent in the next theorem is the same as the one obtained in Theorem 2. 
Theorem 3. Let $n \geq 2$. Assume that $F(K, \cdot)$ satisfies (5) with $Q(K, \cdot)=\rho_{K}^{\theta}, \theta \neq 0$, and that its multipliers satisfy (7). Then, for any $\gamma>0$ and $0<r \leq R$, there is a constant $c_{10}=c_{10}(n, \theta, b, \beta, \gamma, r, R)$ such that

$$
\rho_{2}\left(K, K^{\prime}\right) \leq c_{10}\left\|F(K, \cdot)-F\left(K^{\prime}, \cdot\right)\right\|^{\frac{3}{3+2 \beta}-\gamma}
$$

holds for all $K, K^{\prime} \in \mathcal{K}_{\mathbf{a}, \rho^{\theta}}(r, R)$.

\section{Applications.}

(a) Radial power integrals of central hyperplane sections. Establishing a common framework for different geometrically motivated applications, GROEMER [20] introduces the radial power integral $I_{\theta}^{n}(K)$ of dimension $n$ and order $\theta$ for a convex body $K$ by setting

$$
I_{\theta}^{n}(K)=\int_{S^{n-1}} \rho_{K}^{\theta}(u) d u .
$$

The tomographic data obtained by considering radial power integrals of central hyperplane sections can be written as

$$
F(K, u)=I_{\theta}^{n-1}\left(K \cap u^{\perp}\right)=\left(\mathrm{R} \rho_{K}^{\theta}\right)(u), \quad u \in S^{n-1} .
$$

Theorem 3 can be applied with $\beta=(n-2) / 2$. Hence, for $0<r \leq R, \theta \neq 0$ and $\gamma>0$ there is a constant $c_{11}=c_{11}(n, \theta, \gamma, r, R)$ such that

$$
\rho_{2}\left(K, K^{\prime}\right) \leq c_{11}\left\|I_{\theta}^{n-1}\left(K \cap(\cdot)^{\perp}\right)-I_{\theta}^{n-1}\left(K^{\prime} \cap(\cdot)^{\perp}\right)\right\|^{\frac{3}{n+1}-\gamma}
$$

for all origin-symmetric $K, K^{\prime} \in \mathcal{K}(r, R)$. The same stability result is shown in [20] and [21], but with the generally weaker exponent $2 / n$.

As the $(n-1)$-dimensional volume of the central section with the hyperplane $u^{\perp}$ is

$$
V_{n-1}\left(K \cap u^{\perp}\right)=\frac{1}{n-1} I_{\theta}^{n-1}\left(K \cap u^{\perp}\right), \quad u \in S^{n-1},
$$

(16) can be specialized to

$$
\left.\rho_{2}\left(K, K^{\prime}\right) \leq(n-1) c_{11} \| V_{n-1}\left(K \cap(\cdot)^{\perp}\right)-V_{n-1}\left(K^{\prime} \cap(\cdot)^{\perp}\right)\right) \|^{\frac{3}{n+1}-\gamma},
$$

with the assumptions as above. CAMPI [9, Theorem 1.1] has found the same exponent, generalizing also to bodies that are not necessarily convex, but star shaped with suitable additional properties. Groemer ([21] and [23]) gives also a geometric interpretation of $2 / \varpi_{n} I_{1}^{n}(K)$ as average radial length of $K$ and gives a corresponding stability result with Hölder exponent $2 / n$ which can be improved as above.

(b) Cone-volumes. Let $\Gamma_{\varphi}(u), \varphi \in(0, \pi)$, be a solid cone of revolution with vertex $o$, axis-direction $u$, and fixed opening angle $2 \varphi$. The orientation is here such that $u \in \Gamma_{\varphi}(u)$. Consider the tomographic data obtained as the intersection volumes with such cones:

$$
F(K, u):=V_{n}\left(K \cap \Gamma_{\varphi}(u)\right)=\frac{1}{n}\left(\mathrm{~F}_{\varphi} \rho_{K}^{n}\right)(u), \quad u \in S^{n-1},
$$

where we assumed $o \in K$. If $\varphi$ is a rational multiple of $\pi$ then the multipliers of $F(K, \cdot)$ satisfy (7) with $\beta=(n+2) / 2$. Hence, for $0<r \leq R$, and $\gamma>0$ there is a constant $c_{12}=c_{12}(n, \gamma, r, R)$ such that

$$
\rho_{2}\left(K, K^{\prime}\right) \leq c_{12}\left\|V_{n}\left(K \cap \Gamma_{\varphi}(\cdot)\right)-V_{n}\left(K^{\prime} \cap \Gamma_{\varphi}(\cdot)\right)\right\|^{\frac{3}{n+5}-\gamma}
$$

for all convex bodies $K, K^{\prime} \in \mathcal{K}_{\mathbf{a}, \rho^{\theta}}(r, R)$. In view of the discussion on the spherical cap transform, the Hölder exponent can be improved to $3 /(n+3)-\gamma$ for certain parameter 
values (to be precise, whenever $q r_{0} \notin \mathbb{Z}$ ). For $n=3$ and $\varphi \in\{\pi / 4, \pi / 3\}$, we have $\mathcal{K}_{\mathbf{a}, \theta^{\theta}}(r, R)=\mathcal{K}(r, R)$. The special case $\varphi=\pi / 2$, where the tomographic data consists of half-volumes reads

$$
\rho_{2}\left(K, K^{\prime}\right) \leq c_{12}\left\|V_{n}\left(K \cap(\cdot)^{+}\right)-V_{n}\left(K^{\prime} \cap(\cdot)^{+}\right)\right\|^{\frac{3}{n+3}-\gamma}
$$

for all convex bodies $K, K^{\prime} \in \mathcal{K}(r, R)$ with the property that

$$
\rho_{K}^{n}(u)-\rho_{K}^{n}(-u)=\rho_{K^{\prime}}^{n}(u)-\rho_{K^{\prime}}^{n}(-u)
$$

for all $u \in S^{n-1}$.

(c) Directed section functions and their averages. In analogy to directed projection functions one can define a directed section function $v_{k}(K ; \cdot)$ on $\left\{(L, u) \in \mathcal{L}_{k}^{d} \times S^{n-1}\right.$ : $u \in L\}$ by

$$
v_{k}(K ; L, u)=V_{k}\left(K \cap L \cap u^{+}\right) .
$$

Groemer [23] showed that $v_{n-1}(K ; \cdot)$ determines $K$ uniquely and derived a stability result. GoODEY AND WEIL [14] remark that this implies corresponding uniqueness and stability results for arbitrary $k \geq 2$ with a method of proof like in Application (c) in Section 1.1. (Of course, stability in the case $k=1$ is trivial.) To improve the Hölder exponent we use Theorem 3. Fix $k \in\{2, \ldots, n-1\}, 0<r \leq R, \varepsilon>0, \gamma>0$, and $K, K^{\prime} \in \mathcal{K}(r, R)$ and assume that

$$
\left|v_{k}(K ; L, u)-v_{k}\left(K^{\prime} ; L, u\right)\right| \leq \varepsilon
$$

holds for all $L \in \mathcal{L}_{k}^{n}, u \in S^{n-1} \cap L$. Under these assumptions there is a constant $c_{13}=c_{13}(n, \gamma, r, R)$ such that

$$
\rho_{2}\left(K, K^{\prime}\right) \leq c_{13}\left\|F(K, \cdot)-F\left(K^{\prime}, \cdot\right)\right\|^{\frac{3}{k+2}-\gamma} .
$$

As it appears that the body $K$ is overdetermined by directed section functions, GOODEY AND WEIL [14] consider averaged directed section functions with $k$-dimensional half-planes, $2 \leq k \leq n-1$. Motivated by the stereological concept of vertical sections, they consider

$$
F(K, u):=\int_{\mathcal{L}_{k-1}^{u \perp}} v_{k}(K ; L, u) d L
$$

They show that $F(K, \cdot)$ is an integral transform of the analytical representation $\rho_{K}^{k}$ of $K$. This transform coincides with the one for the average mean directed projection function (11), so injectivity holds for the same pairs $(k, n)$. Due to Theorem 3 , a stability result analogous to (13) is valid, stating that

$$
\rho_{2}\left(K, K^{\prime}\right) \leq c_{13}\left\|F(K, \cdot)-F\left(K^{\prime}, \cdot\right)\right\|^{\frac{3}{2 n+3}-\gamma}
$$

for all $K, K^{\prime} \in \mathcal{K}(r, R)$, where $c_{13}=c_{13}(n, \gamma, r, R)$ is some constant. In view of (26), the Hölder exponent $2 /(n+1)^{2}$ for stability in the Hausdorff metric in [14, Theorem 1.2] can therefore be improved to $6 /((n+1)(2 n+3))-\gamma$.

1.3. Tomographic data from a surface area measure. The following stability results are refinements of those in HUG AND SCHNEIDER [25], which in turn are based on the mentioned work of Bourgain AND Lindenstrauss [5]. With the exception of Theorem 5, results are formulated in terms of the Hausdorff distance. 
Theorem 4. Let $n \geq 2$ and $R \geq r>0$ be given. Assume that $F(K, \cdot)$ satisfies (5) with $Q(K, \cdot)=S_{n-1}(K, \cdot)$ and that its multipliers satisfy $(7)$.

If $\beta<3 / 2$, there is a constant $c_{14}=c_{14}(n, b, \beta, r, R)$ such that

$$
\delta\left(K, K^{\prime}\right) \leq c_{14}\left\|F(K, \cdot)-F\left(K^{\prime}, \cdot\right)\right\|^{\frac{1}{n}}
$$

holds for all $K, K^{\prime} \in \mathcal{K}_{\mathbf{a}, S_{n-1}}(r, R)$.

If $\beta \geq 3 / 2$ and $\gamma>0$, there is a constant $c_{15}=c_{15}(n, b, \beta, \gamma, r, R)$ such that

$$
\delta\left(K, K^{\prime}\right) \leq c_{15}\left\|F(K, \cdot)-F\left(K^{\prime}, \cdot\right)\right\|^{\frac{1}{n(\beta-1 / 2)}-\gamma}
$$

holds for all $K, K^{\prime} \in \mathcal{K}_{\mathbf{a}, S_{n-1}}(r, R)$.

This improves the Hölder exponent $1 /(n(\beta+1))-\gamma$ obtained by Hug AND SchneIDER [25]. We turn to tomographic data depending on surface area measures of lower order $j$ and first treat the simplest case $j=1$. The idea is to reduce the problem to tomographic data depending on support functions using the fact that $S_{1}(K, \cdot)$ is obtained by applying a second order differential operator to $h(K, \cdot)$. As this differential operator is a linear combination of the spherical Laplace-Beltrami operator and the identity, its multipliers are explicitly known.

Theorem 5. Let $n \geq 2$ and $R>0$ be given. Assume that $F(K, \cdot)$ satisfies (5) with $Q(K, \cdot)=S_{1}(K, \cdot)$ and that its multipliers satisfy $(7)$.

If $\beta \leq 2$ then

$$
\delta_{2}\left(K, K^{\prime}\right) \leq c_{16}\left\|F(K, \cdot)-F\left(K^{\prime}, \cdot\right)\right\|
$$

holds for all $K, K^{\prime} \in \mathcal{K}_{\mathbf{a}, S_{1}}(R)$, where $c_{16}=2(n-1) b$.

If $\beta>2$, then, for any $\gamma>0$, there is a constant $c_{17}=c_{17}(n, b, \beta, \gamma, R)$ such that

$$
\delta_{2}\left(K, K^{\prime}\right) \leq c_{17}\left\|F(K, \cdot)-F\left(K^{\prime}, \cdot\right)\right\|^{\frac{3}{2 \beta-1}-\gamma}
$$

holds for all $K, K^{\prime} \in \mathcal{K}_{\mathbf{a}, S_{1}}(R)$.

Hug AND SCHNEIDER [25] also show a stability result for lower order surface area measures in the case where $F(K, \cdot)$ is the cosine transform of $S_{j}(K, \cdot)$. It still holds (with a better exponent) in the general setting, as is shown below.

Theorem 6. Let $n \geq 2$ and $R \geq r>0$ be given. Assume that $F(K, \cdot)$ satisfies (5) with $Q(K, \cdot)=S_{j}(K, \cdot), j \in\{2, \ldots, n-2\}$ and that its multipliers satisfy (7).

If $\beta<3 / 2$, then there is a constant $c_{18}=c_{18}(n, b, \beta, r, R)$ such that

$$
\delta\left(K, K^{\prime}\right) \leq c_{18}\left\|F(K, \cdot)-F\left(K^{\prime}, \cdot\right)\right\|^{\frac{1}{(n+1) 2^{j-1}}}
$$

holds for all $K, K^{\prime} \in \mathcal{K}_{\mathbf{a}, S_{j}}(r, R)$.

If $\beta \geq 3 / 2$ and $\gamma>0$, then there is a constant $c_{19}=c_{19}(n, b, \beta, \gamma, r, R)$ such that

$$
\delta\left(K, K^{\prime}\right) \leq c_{19}\left\|F(K, \cdot)-F\left(K^{\prime}, \cdot\right)\right\|^{\frac{1}{(n+1)(2 \beta-1) 2^{j-2}}-\gamma}
$$

holds for all $K, K^{\prime} \in \mathcal{K}_{\mathbf{a}, S_{j}}(r, R)$.

\section{Applications.}

(a) Illumination and brightness measurements. Motivated by applications in scattering theory, Anikonov And StePAnov [3] consider tomographic data, which is obtained as a linear combination of shadow area and surface area of the "illuminated part" of a convex body,

$$
F(K, u):=a V_{n-1}\left(K \mid u^{\perp}\right)+b S_{n-1}\left(K, S^{n-1} \cap u^{+}\right), \quad u \in S^{n-1} .
$$


They show that $F(K, \cdot)$ determines the convex body $K$ up to translation if and only if the weights $a, b \in \mathbb{R}$ satisfy $a \neq 0 \neq b$ and $a+b \neq 0$. As the integral transforms involved here are the cosine transform and the hemispherical transform, Theorem 4 implies a stability inequality with Hölder exponent arbitrarily close to $2 /(n(n+1))$. A stability result for this data in the case $n=3$ was also derived in [2], but requires that a 6th-order derivative of $F(K, \cdot)$ is uniformly small.

(b) Girth functions and projection functions. Let $V_{j}(K)$ be the $j$-th intrinsic volume of $K, j \in\{1, \ldots, n-1\}$. For the $j$-th order girth function, given by

$$
F(K, u):=V_{j}\left(K \mid u^{\perp}\right)=\frac{1}{2}\left(C S_{j}(K, \cdot)\right)(u), \quad u \in S^{n-1},
$$

we can apply the above theorems with $\beta=(n+2) / 2$. Note that only origin-symmetric convex bodies are determined by one of their girth functions. For $j=n-1$, Theorem 4 leads to the improvement of (6), already discussed in the introduction. For $j=1$, Theorem 5 can be used. The two-dimensional case is special, as $\beta=2$, so

$$
\delta_{2}\left(K, K^{\prime}\right) \leq c_{16}\left\|V_{1}\left(K \mid(\cdot)^{\perp}\right)-V_{1}\left(K^{\prime} \mid(\cdot)^{\perp}\right)\right\|,
$$

which is not amazing, as in the planar case, $1 / 2 V_{1}\left(K \mid u^{\perp}\right)$ is the support function of a rotation of $K$ about the origin with rotation angle $\pi / 2$. For $n \geq 3$, we obtain

$$
\delta_{2}\left(K, K^{\prime}\right) \leq c_{17}\left\|V_{1}\left(K \mid(\cdot)^{\perp}\right)-V_{1}\left(K^{\prime} \mid(\cdot)^{\perp}\right)\right\|^{\frac{3}{n+1}-\gamma}
$$

for all origin-symmetric $K, K^{\prime} \in \mathcal{K}(R)$. This is rephrasing (10). For the cases $2 \leq j \leq$ $n-2$, Theorem 6 yields

$$
\delta\left(K, K^{\prime}\right) \leq c_{19}\left\|V_{j}\left(K \mid(\cdot)^{\perp}\right)-V_{j}\left(K^{\prime} \mid(\cdot)^{\perp}\right)\right\|^{\frac{1}{(n+1)^{2} 2^{j-2}}-\gamma}
$$

for all origin-symmetric $K, K^{\prime} \in \mathcal{K}(r, R)$, improving the Hölder exponent

in [25, Theorem 5.3].

$$
\frac{1}{(n+1)(n+4) 2^{j-2}}-\gamma
$$

It is well known that stability for girth functions implies stability for more general projection functions. Suppose $1 \leq j \leq k \leq n-1$ and assume that there is an $\varepsilon>0$ with

$$
\left|V_{j}(K \mid L)-V_{j}\left(K^{\prime} \mid L\right)\right| \leq \varepsilon
$$

for all $L \in \mathcal{L}_{k}^{n}$. Then Kubota's integral recursion (see e.g. [21, (A.46)]), applied in $u^{\perp}$, gives

$$
\left|V_{j}\left(K \mid u^{\perp}\right)-V_{j}\left(K^{\prime} \mid u^{\perp}\right)\right| \leq c_{19} \varepsilon
$$

for all $u \in S^{n-1}$, where $c_{19}=c_{19}(n, k, j)$ is a constant. The improved Hölder exponents for girth functions therefore extend to general projection functions.

(c) Directed projection functions and their averages. Assume $1 \leq j<k \leq n-1$. The classical (lower order) projection functions, which where mentioned above, can be written as

$$
L \mapsto V_{j}(K \mid L)=c_{j k} S_{j}^{\prime}\left(K \mid L, S^{n-1} \cap L\right) .
$$

Here $c_{j k}=\left(\begin{array}{l}k \\ j\end{array}\right) /\left(k \kappa_{k-j}\right)$ with $\kappa_{m}$ being the volume of the unit ball in $\mathbb{R}^{m}$. The dash indicates that the surface area measure is calculated in the subspace $L$. GOODEY AND WEIL [16] consider directed projection functions

$$
v_{j, k}(K ; L, u)=c_{j k} S_{j}^{\prime}\left(K \mid L, S^{n-1} \cap u^{+}\right)-\frac{c_{j k}}{2} S_{j}^{\prime}\left(K \mid L, S^{n-1} \cap u^{\perp}\right),
$$


yielding the $j$-th order surface area measure of the part of $K \mid L$ which is illuminated in direction $u \in L$. The second term in (20) vanishes for almost all $u \in L$ and the remaining term coincides with the semi-girth of $K$ introduced by GROEMER in [22] for $n=3, k=2$ and $j=1$. For centrally symmetric $K$ the function $2 v_{j, k}(K ; \cdot)$ is independent of $u$ and coincides with $V_{j}(K \mid L)$. The stability result [16, Theorem 2] (which is essentially based on the properties of cosine and hemispherical transform in a fixed but arbitrary $(k+1)$-dimensional subspace) can be improved by modifying its proof. An application of Theorem 6 yields the following slightly strengthened version. Assume that $2 \leq j<k \leq n-1,0<r \leq R, \gamma>0$, and that $K, K^{\prime} \in \mathcal{K}(r, R)$ have coinciding Steiner point. If there is an $\varepsilon>0$ such that, for each $L \in \mathcal{L}_{k}^{n}$ we have

$$
\left|v_{j, k}(K ; L, u)-v_{j, k}\left(K^{\prime} ; L, u\right)\right| \leq \varepsilon
$$

for almost all $u \in S^{n-1} \cap L$, then there is a constant $c_{20}=c_{20}(n, b, \beta, \gamma, r, R)$ such that

$$
\delta\left(K, K^{\prime}\right) \leq c_{20} \varepsilon^{\frac{1}{(k+2)^{2} 2^{j-1}}-\gamma} .
$$

For $j=1$, Theorem 5 can be applied instead and yields an Hölder exponent arbitrarily close to $3 /(k+2)^{2}$.

As convex bodies seem to be overdetermined by directed projection functions, averages are considered in [16], as well. These averages are defined in analogy with the average directed section functions discussed earlier. Besides a general injectivity result for centrally symmetric sets, Goodey and Weil discuss the case $j=1$ in detail, which we treated in Subsection 1.1.

(d) Integrals of sections. For $1 \leq k \leq n-1$, GoOdEY AND WEIL [13] consider the tomographic data

$$
F(K, \cdot)=\int_{\mathcal{L}_{k}^{n}} \int_{L^{\perp}} h(K \cap(L+x), \cdot) d x d L .
$$

The right hand side of (21) is the support function of a convex body which is called mean section body of $K$, as it is derived from the Minkowski average of all $k$-dimensional UIR (uniform isotropic random) planar sections of $K$. For $k=2$ and $n \geq 3$ there is an $x_{K} \in \mathbb{R}^{n}$ with

$$
F(K, \cdot)=\left\langle x_{K}, \cdot\right\rangle+\frac{1}{(n-1) \pi} \int_{S^{n-1}} \Varangle(-v, \cdot) \sin \Varangle(-v, \cdot) S_{n-1}(K, d v)
$$

where $\Varangle(v, u)$ is the smaller angle between the unit vectors $u$ and $v$. From this representation and Theorem 1 it follows that $K \mapsto F(K, \cdot)-\left\langle x_{K}, \cdot\right\rangle$ is a multiplier operator. The multipliers $a_{k}, k \neq 1$, are determined explicitly in [13], showing that they are all nonzero and that (7) is satisfied with $\beta=n$. Theorem 4 implies that for any positive $\gamma, r$ and $R$, there is a constant $c_{21}=c_{21}(n, \gamma, r, R)$ such that

$$
\delta\left(K, K^{\prime}\right) \leq c_{21}\left\|F(K, \cdot)-F\left(K^{\prime}, \cdot\right)\right\|^{\frac{1}{n(n-1 / 2)}-\gamma}
$$

holds for all $K, K^{\prime} \in \mathcal{K}(r, R)$ with Steiner point at the origin. A stability result for this tomographic data was already announced in [13].

(e) Integrated surface area of parallel hyperplane sections. Consider the tomographic measurements

$$
F(K, u):=\frac{1}{2} \int_{-\infty}^{\infty} V_{n-2}\left(K \cap\left(u^{\perp}+t u\right)\right) d t
$$


obtained by invariantly integrating the surface areas of hyperplane sections of $K$ with hyperplanes parallel to $u^{\perp}$. SchNEIDER [30] observed, that

$$
F(K, \cdot)=\frac{1}{n-1} \mathrm{~S}\left(S_{n-1}(K, \cdot)\right)
$$

and thus any full-dimensional origin-symmetric convex body $K$ is uniquely determined by $F(K, \cdot)$. Theorem 4 implies a stability result with Hölder exponent arbitrarily close to $1 /(n(n-1 / 2))$. Motivated by a stereological application and the fact that the odd part of $S_{n-1}(K, \cdot)$ cannot be recovered from $F(K, \cdot)$, SCHNEIDER [33] suggests to restrict surface area measurements to the upper boundary $\partial^{u} K$ of $K$. The set

$$
\partial^{u} K:=\left\{x \in \partial K: \text { there is an outer normal } v \text { to } K \text { at } x \text { with } v \in u^{+}\right\}
$$

can roughly be thought of as the part of the boundary of $K$ that is illuminated by parallel light coming from direction $u$. With $\mathcal{H}^{k}$ denoting $k$-dimensional Hausdorff measure, the new tomographic data can be written as

$$
F(K, u):=\int_{-\infty}^{\infty} \mathcal{H}^{n-2}\left(\partial^{u} K \cap\left(u^{\perp}+t u\right)\right) d t
$$

SCHNEIDER [33] not only shows that

$$
F(K, u)=\int_{S^{n-1} \cap u^{+}} \sqrt{1-\langle v, u\rangle^{2}} S_{n-1}(K, d v),
$$

but also determines the even multipliers of the involved integral transform explicitly and the odd ones approximatively. As they are all non-zero, he concludes that a convex body is uniquely determined up to translation by this tomographic data. His calculations imply that (7) is satisfied for the odd multipliers with $\beta=(n-2) / 2$. By Theorem 4 , a stability result on the class of all convex bodies can only be derived with the same Hölder exponent as obtained for the sine transform. If we restrict to convex bodies of constant brightness, the stability inequality holds with a better Hölder exponent, namely $1 / n$ for $n=3$ and $n=4$, and $2 /(n(n-3))-\gamma$ for $n \geq 5$. Generalizing (22), GOODEY ET AL. [19] consider the upper surface integrals

$$
H_{k}(K, u)=\int_{\mathcal{L}_{k}^{u^{\perp}}} \int_{L^{\perp}} \mathcal{H}^{k-1}\left(\partial^{u} K \cap(L+x)\right) d x d L,
$$

where $1 \leq k \leq n-1$ is fixed. They show that $H_{k}(K, \cdot)$ coincides with a multiple of $H_{n-1}(K, \cdot)$, which is the tomographic data given by $(22)$. Hence, the above stability considerations also hold for $H_{k}(K, \cdot)$.

\section{Notations, KNOWN AND BAsiC RESUlts}

The following notations are used throughout the paper. If not self-explaining or already introduced earlier, detailed definitions will be given later.

$$
\begin{array}{ll}
\langle x, y\rangle & \text { Euclidean scalar product of } x, y \in \mathbb{R}^{n}, n \geq 2, \\
|x| & \text { Euclidean norm of } x \in \mathbb{R}^{n}, \\
B^{n} & =\left\{x \in \mathbb{R}^{n}:|x| \leq 1\right\} \text { unit ball of } \mathbb{R}^{n}, \\
S^{n-1} & =\left\{x \in \mathbb{R}^{n}:|x|=1\right\} \text { unit sphere of } \mathbb{R}^{n}, \\
\mathcal{C} & \text { real Banach space of real continuous functions on } S^{n-1}, \\
& \text { endowed with the maximum norm }\|\cdot\|_{\infty},
\end{array}
$$




$\begin{array}{ll}\mathcal{C}^{m} & m \text {-times continuously differentiable functions in } \mathcal{C}, \\ & m \in \mathbb{N}_{0},\left(\text { with } \mathcal{C}^{0}=\mathcal{C}\right), \\ & \text { real Banach space of } p \text {-integrable real functions on } S^{n-1} \text { with } \\ & \text { norm }\|\cdot\|_{p}, \\ \langle\cdot, \cdot\rangle \text { and }\|\cdot\| \quad & \text { natural scalar product and norm in } L^{2} \text { (hence }\|\cdot\|=\|\cdot\|_{2} \text { ), } \\ & \text { Sobolev space of } L^{2} \text {-functions with derivatives up to order } \\ & \eta \geq 0, \\ & \text { natural norm in } H^{\eta}, \\ \|\cdot\|_{H^{\eta}} & \text { space of spherical harmonics of dimension } n \text { and degree } k, \\ \mathcal{H}_{k}^{n} & \text { Legendre polynomial of dimension } n \text { and degree } k, \\ P_{k}^{n}(\cdot) & \text { real vector space of finite signed measures on the sphere } S^{n-1} \\ \mathcal{M} & \text { endowed with the weak topology, } \\ & \text { proper orthogonal group of } \mathbb{R}^{n}, \\ S O_{n} & \text { family of convex bodies in } \mathbb{R}^{n}, \\ \mathcal{K} & \text { Hausdorff distance and } L^{2} \text {-distance of } K \text { and } K^{\prime} \text { in } \mathcal{K}, \\ \delta\left(K, K^{\prime}\right), \delta_{2}\left(K, K^{\prime}\right) & \text { radial } L^{2} \text {-distance of } K \text { and } K^{\prime} \text { in } \mathcal{K}, \\ \rho_{2}\left(K, K^{\prime}\right) & j \text {-th intrinsic volume of } K \in \mathcal{K}, j \in\{0, \ldots, n\}, \\ V_{j}(K) & \text { support function of } K \in \mathcal{K}, \\ h_{K} & \text { radial function of } K \in \mathcal{K}, \\ \rho_{K} & j \text {-th surface area measure of } K \in \mathcal{K}, j \in\{0, \ldots, n-1\} . \\ S_{j}(K, \cdot) & \end{array}$

The surface area of the unit sphere $S^{n-1}$ in $\mathbb{R}^{n}$ is denoted by $\varpi_{n}$, the volume of the Euclidean unit ball $B^{n}$ by $\kappa_{n}$. The spaces $L^{p}, 1 \leq p \leq \infty$ are consisting of all functions $f: S^{n-1} \rightarrow \mathbb{R}$ which are $p$-integrable with respect to spherical Lebesgue measure (with the usual meaning when $p=\infty$ ). The space $L^{2}$ of square integrable functions on the sphere is endowed with the scalar product

$$
\langle\langle f, g\rangle\rangle=\int_{S^{n-1}} f(u) g(u) d u . \quad f, g \in L^{2},
$$

and the derived norm $\|\cdot\|$. The rotation of a function $f \in L^{1}$ is defined by

$$
\vartheta f(u)=f\left(\vartheta^{-1} u\right), \quad u \in S^{n-1}, \vartheta \in S O_{n} .
$$

The non-empty compact convex subsets of $\mathbb{R}^{n}$ are called convex bodies and the family of all convex bodies is denoted by $\mathcal{K}$. The support function

$$
h_{K}=\max \{\langle x, \cdot\rangle: x \in K\}
$$

of $K \in \mathcal{K}$ is considered as a function on $S^{n-1}$, if not otherwise stated. The Hausdorff metric on $\mathcal{K}$ can conveniently be defined by

$$
\delta\left(K, K^{\prime}\right)=\left\|h_{K}-h_{K^{\prime}}\right\|_{\infty}, \quad K, K^{\prime} \in \mathcal{K} .
$$

The $L^{2}$-metric on $\mathcal{K}$, defined in Section 1.1, is equivalent to the Hausdorff metric: for $K, K^{\prime} \in \mathcal{K}(R)$ we have

$$
\varpi_{n}^{-1 / 2} \delta_{2}\left(K, K^{\prime}\right) \leq \delta\left(K, K^{\prime}\right) \leq c_{22} \delta_{2}\left(K, K^{\prime}\right)^{2 /(n+1)},
$$


where

$$
c_{22}=\left(\frac{(n+1) n(n-1)}{2 \varpi_{n-1}}\right)^{1 /(n+1)} R^{(n-1) /(n+1)}
$$

(see e.g. [21, Proposition 2.3.1]). The mean value theorem implies that

$$
\delta_{2}\left(K, K^{\prime}\right) \leq c_{23}\left\|h_{K}^{\theta}-h_{K^{\prime}}^{\theta}\right\|
$$

holds for all $K, K^{\prime} \in \mathcal{K}(r, R)$ and $\theta \neq 0$, with

$$
c_{23}=|\theta|^{-1}\left(r^{1-\theta} \vee R^{1-\theta}\right) \text {. }
$$

We note similar results for the radial $L^{2}$-distance. If $K, K^{\prime} \in \mathcal{K}(r, R)$ then

$$
\rho_{2}\left(K, K^{\prime}\right) \leq c_{23}\left\|\rho_{K}^{\theta}-\rho_{K^{\prime}}^{\theta}\right\|
$$

and

$$
\delta\left(K, K^{\prime}\right) \leq c_{24} \rho_{2}\left(K, K^{\prime}\right)^{2 /(n+1)} ;
$$

see [21, Lemma 2.3.2], also for an explicit value of $c_{24}=c_{24}(n, r, R)$.

If not otherwise stated, the following facts about spherical harmonic analysis can be found in Groemer's book [21]. Let $\mathcal{H}_{k}^{n}$ be the space of spherical harmonics of order $k$ and dimension $n$. Fix $u \in S^{n-1}$. Up to multiplication with a constant, there is exactly one spherical harmonic $Y_{k} \in \mathcal{H}_{k}^{n}$, which is zonal with respect to $u$ (i.e. it is invariant under all rotations at the origin fixing $u$ ). The additional normalizing condition $Y_{k}(u)=1$ implies $Y_{k}=P_{k}^{n}(\langle u, \cdot\rangle)$, where $P_{k}^{n}$ is the Legendre polynomial of degree $k$ and dimension $n$. The (condensed) spherical harmonic expansion of an integrable function $f$ on the sphere is denoted by $f \sim \sum_{k=0}^{\infty} f_{k}$, where

$$
f_{k}=c(k) \int_{S^{n-1}} f(u) P_{k}^{n}(\langle u, \cdot\rangle) d u,
$$

with $c(k)=\operatorname{dim} \mathcal{H}_{k}^{n} / \varpi_{n}$. Let $\mathcal{M}$ be the vector space of finite signed measures on the unit sphere, endowed with the weak topology. We will also work with the (formal) spherical harmonic expansion of signed measures $\mu \in \mathcal{M}$, writing $\mu \sim \sum_{k=0}^{\infty} \mu_{k}$, where

$$
\mu_{k}=c(k) \int_{S^{n-1}} P_{k}^{n}(\langle u, \cdot\rangle) \mu(d u)
$$

For $f \in \mathcal{C}^{\infty}$ and $\mu \in \mathcal{M}$ with spherical harmonic expansions $f \sim \sum_{k=0}^{\infty} f_{k}$ and $\mu \sim$ $\sum_{k=0}^{\infty} \mu_{k}$, respectively, the generalization of Parseval's identity

$$
\int_{S^{n-1}} f d \mu=\sum_{k=0}^{\infty}\left\langle\left\langle f_{k}, \mu_{k}\right\rangle\right\rangle
$$

holds. Due to (27), the spherical harmonic expansion of $\mu \in \mathcal{M}$ determines this measure uniquely. A more general discussion of spherical harmonic expansions for distributions (i.e. generalized functions) on the sphere can be found in [27].

Derivatives of a function $f$ on the sphere are understood as derivatives of its homogeneous extension of degree zero. In particular $\nabla_{0} f$ is the gradient of this extension, restricted to $S^{n-1}$ and $\Delta_{0}$ is the spherical Laplace-Beltrami operator. For every $k=0,1,2, \ldots$, the set $\mathcal{H}_{k}^{n}$ is an eigenspace of $\Delta_{0}$ and the corresponding eigenvalue (or multiplier) is $-k(k+n-2)$. Let $\mathcal{C}^{m} \subset \mathcal{C}, m \in \mathbb{N}$, be the real vector space of all $m$-times continuously differentiable functions. Convergence in this space is understood 
as uniform convergence of all derivatives up to order $m$. Set $\mathcal{C}^{\infty}=\bigcap_{m=0}^{\infty} \mathcal{C}^{m}$. If $f \in \mathcal{C}^{2}$, then Green's formula and Parseval's equality imply

$$
\left\|\left|\nabla_{0} f\right|\right\|^{2}=-\left\langle\left\langle f, \Delta_{0} f\right\rangle=\sum_{k=0}^{\infty} k(k+n-2)\left\|f_{k}\right\|^{2} .\right.
$$

We will also need fractional derivatives of spherical functions. For $\eta \geq 0$ we define the Sobolev space $H^{\eta}$ as the family of those square integrable functions $f \sim \sum_{k=0}^{\infty} f_{k}$ for which

$$
\|f\|_{H^{\eta}}^{2}:=\sum_{k=0}^{\infty}(1 \vee k)^{2 \eta}\left\|f_{k}\right\|^{2}
$$

is finite. The space $H^{\eta}$ is a Banach space with norm $\|\cdot\|_{H^{\eta}}$. In view of the eigenvalues of the Laplace-Beltrami operator, Campi [10] introduces the same space considering functions $f \in L^{2}$ for which

$$
\sum_{k=0}^{\infty}(k(k+n-2))^{\eta}\left\|f_{k}\right\|^{2}
$$

converges. The convergence of this sum implies that

$$
\sum_{k=0}^{\infty}(k(k+n-2))^{\eta / 2} f_{k}
$$

converges in the $L^{2}$-sense to a function, which is denoted by $\left(-\Delta_{0}\right)^{\eta / 2} f$. We have, of course, $H^{0}=L^{2}$. CAMPI $\left[9\right.$, Lemma 4.1] shows that if $0<\eta<3 / 2,\left|\nabla_{0} f\right| \in L^{\infty}$, and $\Delta_{0} f \in L^{1}$, then

$$
\left\|\left(-\Delta_{0}\right)^{\eta / 2} f\right\|^{2} \leq c_{25}\left\|\left|\nabla_{0} f\right|\right\|_{\infty}\left\|\Delta_{0} f\right\|_{1}
$$

for some constant $c_{25}=c_{25}(n, \eta)$, and hence $f \in H^{\eta}$. We note that Parseval's equality implies

$$
\left\langle\left\langle f,\left(-\Delta_{0}\right)^{\eta / 2} g\right\rangle=\left\langle\left\langle\left(-\Delta_{0}\right)^{\eta / 2} f, g\right\rangle\right\rangle\right.
$$

for $f, g \in H^{\eta}$, which generalizes the well-known self-adjointness of $\Delta_{0}$.

The above concepts can in particular be applied to powers of support functions and radial functions. Let $K$ be a convex body and $h_{K} \sim \sum_{k=0}^{\infty} h_{k}$ be the spherical harmonic expansion of its support function. Then the constant $2 h_{0}$ coincides with the mean width $\bar{w}(K)$ of $K$ and $h_{1}=\langle s(K), \cdot\rangle$ is the support function of a singleton containing the Steiner point $s(K)$ of $K$. We mention an important connection between support functions and first order surface area measures. BERG [4] showed that

$$
S_{1}(K, \cdot)=\square h_{K}
$$

in the sense of distributions, where

$$
\square=\frac{\Delta_{0}}{n-1}+1
$$

is a second order differential operator. Note that $\square$ acts as a multiple $\lambda_{k}$ of the identity on $\mathcal{H}_{k}^{n}$, where

$$
\lambda_{k}=-\frac{(k-1)(k+n-1)}{n-1}, \quad k=0,1,2, \ldots
$$

Let $K \subset R B^{n}, R>0$, be strictly convex and $x(u)$ be the point in bd $K$ with outer unit normal $u \in S^{n-1}$. Then, considering $h_{K}$ as a (positive homogeneous) function 
on $\mathbb{R}^{n}, \nabla h_{K}(u)=\langle x(u), \cdot\rangle$ and thus $\left\|\left|\nabla h_{K}\right|\right\|_{\infty} \leq R$. Using the fact that $h_{K}(\cdot)$ is homogeneous of degree one, this relation can be transferred to $\nabla_{0} h_{K}$ and yields

$$
\left\|\left|\nabla_{0} h_{K}\right|\right\|_{\infty} \leq 2 R
$$

This relation also holds without the assumption of strict convexity. If $K$ has a smooth boundary, then $\square h_{k}=S_{1}(K, \cdot)$ is a nonnegative function, and thus

$$
-(n-1) h_{K} \leq \Delta_{0} h_{K}
$$

The last inequality, together with the additional assumption $o \in K$ implies $\left|\Delta_{0} h_{K}\right| \leq$ $\Delta_{0} h_{K}+(n-1) h_{K}$. Integrating this, using also (29) with $\eta=2$, gives

$$
\left\|\Delta_{0} h_{K}\right\|_{1} \leq(n-1) \varpi_{n} R \text {. }
$$

Substituting (32) and (33) into (28) shows

$$
\left\|\left(-\Delta_{0}\right)^{\eta / 2} h_{K}\right\| \leq c_{26},
$$

where $0<\eta<3 / 2$ and $c_{26}=c_{26}(n, \eta, R)$ is independent of $K \subset R B^{n}$. An approximation argument shows that (34) is true even without the smoothness assumptions on $K$. Also, the assumption $o \in K$ can obviously be dropped possibly increasing the constant $c_{26}$. In particular, $h_{K} \in H^{\eta}$ for any positive $\eta<3 / 2$. (34) was shown by CAMPI [8] in the case $n=3$. We generalize these observations slightly.

Proposition 7. Fix $0<r \leq R, 0<\eta<3 / 2$. Then the following sets are bounded in $H^{\eta}$ :

(a) $\left\{h_{K}^{\theta}: K \in \mathcal{K}(r, R)\right\}$ for $\theta \in \mathbb{R}$,

(b) $\left\{h_{K}^{\theta}: K \in \mathcal{K}(0, R)\right\}$ for $\theta \geq 2$,

(c) $\left\{h_{K}^{\theta}: K \in \mathcal{K}(R)\right\}$ for $\theta \in \mathbb{N}$.

Proof. For $\theta=1$ the estimate (34) implies the strongest claim (c), so we may assume $\theta \notin\{0,1\}$. For this proof only let $\hat{f}$ be positive homogeneous extension of degree zero of the spherical function $f$. We have

$$
\nabla \widehat{\left(f^{\theta}\right)}=\nabla(\widehat{f})^{\theta}=\theta(\widehat{f})^{\theta-1} \nabla \widehat{f}
$$

and

$$
\Delta \widehat{\left(f^{\theta}\right)}=\theta \widehat{f}^{\theta-2}\left((\theta-1)|\nabla \widehat{f}|^{2}+\widehat{f} \Delta \widehat{f}\right) .
$$

For $f=h_{K}$ with $K \in \mathcal{K}$ and $r B^{n} \subset K \subset R B^{n}$, (32) and (33) give

$$
\begin{aligned}
\left\|\left|\nabla_{0} h_{K}^{\theta}\right|\right\|_{\infty} & =\left\|\left|\theta\left(h_{K}\right)^{\theta-1}(u) \nabla_{0} h_{K}(u)\right|\right\|_{\infty} \\
& \leq|\theta|\left(r^{\theta-1} \vee R^{\theta-1}\right)\left\|\left|\nabla_{0} h_{K}(u)\right|\right\|_{\infty} \leq c_{27}(n, \theta, r, R)
\end{aligned}
$$

and

$$
\left\|\Delta_{0} h_{K}^{\theta}\right\|_{1} \leq|\theta|\left(r^{\theta-2} \vee R^{\theta-2}\right) \quad\left\|(\theta-1)\left|\nabla_{0} h_{K}\right|^{2}+h_{K} \Delta_{0} h_{K}\right\|_{1} \leq c_{28}(n, \theta, r, R) .
$$

The claim (a) now follows from (28). If $\theta \geq 2$ then $r^{\theta-1} \vee R^{\theta-1}=R^{\theta-1}$ and $r^{\theta-2} \vee R^{\theta-2}=$ $R^{\theta-2}$ are independent of $r$, so we may replace the condition $r B^{n} \subset K$ by $o \in K$ (i.e. $h_{K} \geq 0$ ) when $\theta$ is not an integer, and omit it completely for $\theta \in \mathbb{N}$.

Similar results hold for powers of the radial function of a convex body. If $K^{*}$ denotes the polar body of $K$ (with the origin $o$ in the interior), then $\rho_{K}^{-1}=h_{K^{*}}$ and $r B^{n} \subset K \subset$ $R B^{n}$ implies $1 / R B^{n} \subset K^{*} \subset 1 / r B^{n}$. This gives the following result. 
Corollary 8. Fix $0<r \leq R, 0<\eta<3 / 2$, and $\theta \in \mathbb{R}$. Then

$$
\left\{\rho_{K}^{\theta}: K \in \mathcal{K}(r, R)\right\}
$$

is a bounded set in $H^{\eta}$.

CAmpi [10, Theorem 2.4 and Lemma 2.5] showed Corollary 8 for the case $\theta=1$. His proof already contains all essential arguments for the general case.

\section{Proofs AND AUXiliary RESUlts}

3.1. Proof of Theorem 1. A first step to prove Theorem 1 is to show that any sufficiently smooth function can be written as a linear combination of $Q(K, \cdot)$ with coefficients in $\{ \pm 1\}$. The next two Propositions provide this result for the different choices of $Q(K, \cdot)$.

Proposition 9. Let $\theta \neq 0$. For any $f \in \mathcal{C}^{2}$ there is a convex body $K$ with the origin in its interior, and an $r>0$ such that

$$
f=h_{K}^{\theta}-h_{r B^{n}}^{\theta}
$$

Here, $K$ and $r$ can be chosen in a continuous way, i.e. there is a mapping $f \mapsto(K, r)$, continuous from $\mathcal{C}^{2}$ to $\mathcal{K} \times \mathbb{R}_{>0}$, such that (35) holds.

Likewise, there is a convex body $M$ with the origin in its interior and an $r>0$ such that

$$
f=\rho_{M}^{\theta}-\rho_{r B^{n}}^{\theta}
$$

Here, $M$ and $r$ can be chosen in a continuous way, i.e. there is a mapping $f \mapsto(M, r)$, continuous from $\mathcal{C}^{2}$ to $\mathcal{K} \times \mathbb{R}_{>0}$, such that (36) holds.

Proof. For $\theta=1$, the claim was shown by SchneIder [31] (cf. also [27, p. 5555]). His arguments can be extended to the case $\theta \notin\{0,1\}$ as follows. To start, consider only functions $f \in \mathcal{C}^{2}$ which are positive on $S^{n-1}$. Let $\check{f}$ be the positive homogeneous extension of degree one of $f^{1 / \theta}$. For $s \geq 0$, the function

$$
g(x)=\left(\|x\|^{\theta}+s(\check{f})^{\theta}(x)\right)^{1 / \theta}, \quad x \neq o,
$$

has gradient

$$
\nabla g(x)=g^{1-\theta}(x)\left(\|x\|^{\theta-2} x+s(\check{f})^{\theta-1}(x) \nabla \check{f}(x)\right) .
$$

The matrix of its second derivatives (at a point $u \in S^{n-1}$ ) therefore is given by

$$
\begin{aligned}
H_{g}(u)= & (1-\theta) g^{-1}(u) \nabla g(u)(\nabla g(u))^{\top}+g^{1-\theta}(u)\left[I+(\theta-2) u u^{\top}\right. \\
& \left.+s(\theta-1) \check{f}^{\theta-2}(u)(\nabla \check{f})(u)((\nabla \check{f})(u))^{\top}+s \check{f}^{\theta-1}(u) H_{\check{f}}(u)\right],
\end{aligned}
$$

where $I$ is the identity matrix and $H_{\breve{f}}$ is the matrix of second derivatives of $\check{f}$. It is well known that $g$ is the support function of a convex body if and only if this matrix is positive semi-definite for all $u \in S^{n-1}$. Fix $u \in S^{n-1}$. As $g$ is positive homogeneous of degree one, $u$ is an eigenvector of $H_{g}(u)$ with corresponding eigenvalue 0 . It is therefore enough to control $y^{\top} H_{g}(u) y$ for $y \in S^{n-1} \cap u^{\perp}$. If $y$ is a unit vector orthogonal to $u$, then

$$
y^{\top} \nabla g(u)(\nabla g(u))^{\top} y=s^{2} g^{2(1-\theta)}(u) \check{f}^{2(\theta-1)}(u)\langle\nabla \check{f}(u), y\rangle^{2},
$$


and thus

$$
\begin{aligned}
y^{\top} H_{g}(u) y=g^{1-\theta}(u)[ & 1+s \check{f}^{\theta-1}(u) y^{\top} H_{\check{f}}(u) y \\
& \left.+s(\theta-1) \check{f}^{\theta-2}(u)\langle\nabla \check{f}(u), y\rangle^{2}\left(1-s(\check{f}(u) / g(u))^{\theta}\right)\right] .
\end{aligned}
$$

As $0<1-s(\check{f}(u) / g(u))^{\theta} \leq 1$, the last summand is not smaller than $-1 / 2$, whenever

$$
s \leq s_{1}(u):=\frac{1}{2}|1-\theta|^{-1} f^{2-\theta}(u)|\nabla \check{f}(u)|^{-2} .
$$

Let $\eta_{\check{f}}(u)$ be the smallest eigenvalue of $H_{\check{f}}(u)$ and let

$$
s \leq s_{2}(u):= \begin{cases}\infty, & \text { if } \eta_{\check{f}}(u) \geq 0, \\ -\frac{1}{2 \eta_{\check{f}}(u)} f^{1-\theta}(u), & \text { if } \eta_{\check{f}}(u)<0 .\end{cases}
$$

Then $s \leq \min \left\{s_{1}(u), s_{2}(u)\right\}$ implies that $H_{g}(u)$ is positive semi-definite. Setting

$$
s_{i}=\min _{u \in S^{n-1}} s_{i}(u), \quad i=1,2,
$$

and $\rho=\min \left\{s_{1}, s_{2}\right\}>0$ we see that

$$
g_{\rho}(x)=\left(\|x\|^{\theta}+\rho(\check{f})^{\theta}(x)\right)^{1 / \theta}, \quad x \neq o,
$$

is the support function of a convex body $N$. As $h_{N}=g_{\rho}>0$ on the unit sphere, $o$ is an interior point of $N$ and

on $S^{n-1}$. We obtain

$$
f=\rho^{-1} h_{N}^{\theta}-\rho^{-1}
$$

$$
f=h_{K}^{\theta}-h_{r B^{n}}^{\theta}
$$

with $r:=\rho^{-1 / \theta}$ and $K:=\rho^{-1 / \theta} N$. The functions $s_{1}(u)$ and $s_{2}(u)$ depend continuously on $f$ and so do $\rho$ and $r$. This also implies the continuity of $f \mapsto(1 / \rho+f)^{1 / \theta}=h_{K}$. Hence $K$ depends continuously on $f$. Summarizing, we have shown (35) and the corresponding continuity statement for the family of all positive functions $f \in \mathcal{C}^{2}$. The positivity condition is here not essential, as we can apply the above arguments to the function $f+1+\|f\|_{\infty}$ and use the fact that the constant $1+\|f\|_{\infty}$ only changes the radius $r$ in the representation of $f$.

The second claim is an easy consequence of the above and polar duality, using again that $\rho_{K^{*}}=h_{K}^{-1}$. Applying (35) with $\theta$ replacing $-\theta$ gives

$$
f=h_{K}^{-\theta}-h_{r B^{n}}^{-\theta}=\rho_{K^{*}}^{\theta}-\rho_{1 / r B^{n}}^{\theta}
$$

for a suitable convex body $K$ with $o$ in its interior and $r>0$. This shows (36). The continuity of $K \mapsto K^{*}$ on the class of convex bodies (with $o$ as interior point) implies the continuity statement and the proof is complete.

For $m \in \mathbb{N}_{0}$, let $\mathcal{C}_{*}^{m}$ be the space of of all functions $f \in \mathcal{C}^{m}$ which are centered in the sense that

$$
f_{1}=c(1) \int_{S^{n-1}}\langle u, \cdot\rangle f(u) d u \equiv 0 .
$$

Proposition 10. Let $\mathcal{V}_{j}$ be the linear subspace of $\mathcal{M}$ spanned by the cone $\left\{S_{j}(K, \cdot)\right.$ : $\left.K \in \mathcal{K}_{0}\right\}, j \in\{1, \ldots, n-1\}$. Then, for any $j \in\{1, \ldots, n-1\}$,

$$
\mathcal{C}_{*}^{2\lceil(n+1) / 4\rceil} \subset \mathcal{V}_{1} \subset \mathcal{V}_{j}
$$

and there is a continuous mapping

$$
f \mapsto\left(K_{1}, \ldots, K_{j}, r\right)
$$


from $\mathcal{C}_{*}^{2\lceil(n+1) / 4\rceil}$ to $\mathcal{K}^{j} \times \mathbb{R}_{>0}$ such that

$$
f=\sum_{i=1}^{j} S_{j}\left(K_{i}, \cdot\right)-S_{j}\left(r B^{n}, \cdot\right) .
$$

Remark 11. Like in the case $j=1$, also when $j=n-1$, any $f \in \mathcal{C}_{*}^{\infty}$ can be written as

$$
f=S_{j}(K, \cdot)-S_{j}\left(r B^{n}, \cdot\right)
$$

(with a suitable convex body $K$ and $r>0$ ), as $\mathcal{V}_{n-1}$ is closed under addition. This is not true for $2 \leq j \leq n-2$. The proof of Proposition 10 is a refinement of WEIL's proof in [40, Theorem 3.5], where it is shown that differences of $j$-th order surface area measures are dense in the set $\mathcal{M}_{*}:=\left\{\mu \in \mathcal{M} \mid \mu_{1}=o\right\}$ of all signed measures with centroid at the origin.

Proof. Assume $f \in \mathcal{C}_{*}^{2\lceil(n+1) / 4\rceil}$ with $f \sim \sum_{k=0}^{\infty} f_{k}$, and let $\lambda_{k}$ be given by (31). The sum

$$
\sum_{k \neq 1} \lambda_{k}^{-1} f_{k}
$$

converges uniformly to a function $h_{f} \in \mathcal{C}_{*}^{2}$ and the mapping $f \mapsto h_{f}$ from $\mathcal{C}_{*}^{2\lceil(n+1) / 4\rceil}$ to $\mathcal{C}_{*}^{2}$ is continuous. This follows from SeELEY [36, Theorem 4(b)]; cf. [27, p. 5549] for a similar reasoning. As $\lambda_{0}, \lambda_{1}, \ldots$ are the multipliers of $\square$, we have $\square h_{f}=f$. There is a convex body $K$ and an $r>0$, both continuously depending on $h_{f}$, with $h_{f}=h_{K}-h_{r B^{n}}$ by Proposition 9, and (30) implies

$$
f=\square h_{f}=S_{1}(K, \cdot)-S_{1}\left(r B^{n}, \cdot\right) \in \mathcal{V}_{1} .
$$

The pair $(K, r)$ constructed this way depends continuously on $f$ and the proposition is shown for $j=1$.

To show the claim for $2 \leq j \leq n-1$ we start by fixing $K \in \mathcal{K}$ and show that there is a linear combination of the $j$-th surface area measures of

$$
K, K+B^{n}, K+2 B^{n}, \ldots, K+(j-1) B^{n}
$$

which coincides with $S_{1}(K, \cdot)$ (up to addition of a suitable multiple of spherical Lebesgue measure). Consider the linear combination

$$
\mu_{j}(K, \cdot)=\frac{1}{j !} \sum_{s=0}^{j-1}\left(\begin{array}{c}
j-1 \\
s
\end{array}\right)(-1)^{j-1-s} S_{j}\left(K+s B^{n}, \cdot\right) \in \mathcal{V}_{j}
$$

Using that

$$
S_{j}\left(K+s B^{n}, \cdot\right)=\sum_{i=0}^{j}\left(\begin{array}{l}
j \\
i
\end{array}\right) s^{j-i} S_{i}(K, \cdot)
$$

we obtain

$$
\mu_{j}(K, \cdot)=\sum_{i=0}^{j} b(i) S_{i}(K, \cdot)
$$

where the coefficients are given by

$$
b(i)=\frac{1}{j !}\left(\begin{array}{l}
j \\
i
\end{array}\right) \sum_{s=0}^{j-1}\left(\begin{array}{c}
j-1 \\
s
\end{array}\right)(-1)^{j-1-s} s^{j-i} .
$$


From $[24$, p. $64,(x x i)]$, it follows $b(1)=1$ and $b(t)=0$ for all $2 \leq t \leq j$. The constant $b(0)=(j-1) / 2$ is not explicitly needed in what follows, but we give it for the reader's convenience. We obtain

$$
\mu_{j}(K, \cdot)=S_{1}(K, \cdot)+\frac{j-1}{2} S_{0}\left(B^{n}, \cdot\right),
$$

as $S_{0}(K, \cdot)=S_{0}\left(B^{n}, \cdot\right)$. Observing that for any $\mu \in \mathcal{V}_{1}$, there are $K$ and $K^{\prime}$ in $\mathcal{K}$ such that

$$
\mu=S_{1}(K, \cdot)-S_{1}\left(K^{\prime}, \cdot\right)=\mu_{j}(K, \cdot)-\mu_{j}\left(K^{\prime}, \cdot\right) \in \mathcal{V}_{j},
$$

we see $\mathcal{V}_{1} \subset \mathcal{V}_{j}$

To construct the continuous mapping $f \mapsto\left(K_{1}, \ldots, K_{j}, r\right)$ we use the proposition for the case $j=1$, which has already been treated: There is a continuous mapping $f \mapsto(K, \rho)$ such that

$$
f=S_{1}(K, \cdot)-S_{1}\left(\rho B^{n}, \cdot\right) .
$$

Obviously, $r:=((j-1) / 2+\rho)^{1 / j}$ and

$$
K_{i}:=\left(\frac{1}{j !}\left(\begin{array}{c}
j-1 \\
i-1
\end{array}\right)\right)^{1 / j}\left(K+(i-1) B^{n}\right), \quad i=1, \ldots, j,
$$

depend continuously on $f$ and we obtain from $(38)$ and $S_{j}\left(B^{n}, \cdot\right)=S_{0}\left(B^{n}, \cdot\right)$

$$
\begin{aligned}
\sum_{i=1}^{j} S_{j}\left(K_{i}, \cdot\right)-S_{j}\left(r B^{n}, \cdot\right) & =\mu_{j}(K, \cdot)-\frac{j-1}{2} S_{0}\left(B^{n}, \cdot\right)-\rho S_{1}\left(B^{n}, \cdot\right) \\
& =S_{1}(K, \cdot)-S_{1}\left(\rho B^{n}, \cdot\right)=f .
\end{aligned}
$$

Lemma 12. Let $\theta \neq 0$ and assume that $\mathcal{D}$ is one of the (abstract) cones $\left\{h_{K}^{\theta}: K \in\right.$ $\mathcal{K}, o \in K\},\left\{\rho_{K}^{\theta}: K \in \mathcal{K}, o \in K\right\}$ (supplied with the topology induced by the maximum norm) and $\left\{S_{j}(K, \cdot): K \in \mathcal{K}\right\}$ (supplied with the weak topology). Then $\mathcal{D} \cap \mathcal{C}^{\infty}$ is dense in $\mathcal{D}$.

Proof. We distinguish the different cases:

(a) $\mathcal{D}=\left\{h_{K}^{\theta}: K \in \mathcal{K}, o \in K\right\}$. The case $\theta=1$ is well known (cf. Schneider [32, Theorem 3.3.1] and trivially implies the general case.

(b) For $\mathcal{D}=\left\{\rho_{K}^{\theta}: K \in \mathcal{K}, o \in K\right\}$, we may restrict considerations to convex bodies $K$ with $o \in \operatorname{int} K$ and the claim follows from (a) by polar duality.

(c) Fix $K \in \mathcal{K}$. According to (a), there is a sequence $\left(K_{i}\right)$ of convex bodies with $\mathcal{C}^{\infty}$ support functions and $\lim _{i \rightarrow \infty} K_{i}=K$. By [32, Corollary 2.5.3], $S_{j}\left(K_{i}, \cdot\right)$ has a $\mathcal{C}^{\infty}$ density for $i=1,2,3, \ldots$ and the claim follows from the weak continuity of $K \mapsto S_{j}(K, \cdot)$.

Proof of Theorem 1. Assume that $F$ satisfies (i) - (iv) and set

$$
\mathcal{V}=\left\{\sum_{i=1}^{k} \varepsilon_{i} Q\left(K_{i}, \cdot\right): K_{1}, \ldots, K_{k} \in \mathcal{K}, \varepsilon_{1}, \ldots, \varepsilon_{k} \in\{ \pm 1\}, k \in \mathbb{N}\right\} .
$$

By (iv), the mapping $T: \mathcal{V} \rightarrow L^{2}$, given by

$$
T f=\sum_{i=1}^{k} \varepsilon_{i} F\left(K_{i}, \cdot\right)
$$


with $f=\sum_{i=1}^{k} \varepsilon_{i} Q\left(K_{i}, \cdot\right) \in \mathcal{V}$ is well defined and linear. Due to (iii), $T$ commutes with rotations. Propositions 9 and 10 imply that $\mathcal{C}^{2} \subset \mathcal{V}$ and $\mathcal{C}_{*}^{m} \subset \mathcal{V}, m=2\lceil(n+1) / 4\rceil$, depending on the choice of $Q(K, \cdot)$. When $Q(K, \cdot)$ is a surface area measure, we define

$$
T f:=T\left(f-f_{1}\right), \quad f \in \mathcal{C}^{m},
$$

and thus, $T$ is defined on all of $\mathcal{C}^{m}$, independent of the analytical representation. By an application of Schur's Lemma (cf. [31, p. 67] in a similar situation), it follows that for any $k \in \mathbb{N}_{0}$, there is $a_{k} \in \mathbb{R}$ and a $\vartheta_{k} \in S O_{n}$ (with $\vartheta_{k}$ being the identity when $n \geq 3$ ) such that

$$
T f=a_{k} \vartheta_{k} f, \quad f \in \mathcal{H}_{k}^{n} .
$$

Here $a_{1}=0$, when $Q(K, \cdot)$ is a surface area measure.

The continuity statements in Propositions 9 and 10 and (ii) imply that $T: \mathcal{C}^{m} \rightarrow L^{2}$ is continuous. If $f \in \mathcal{C}^{\infty}$ with $f \sim \sum_{k=0}^{\infty} f_{k}$, then $\sum_{k=0}^{\infty} f_{k}$ converges to $f$ in $\mathcal{C}^{m}$ and (39) implies

$$
T f \sim \sum_{k=0}^{\infty} a_{k} \vartheta_{k} f_{k} .
$$

In particular, if $f=Q(K, \cdot)$ is in $\mathcal{C}^{\infty}$, we may use $F(K, \cdot)=T Q(K, \cdot)$ and obtain

$$
Q(K, \cdot) \sim \sum_{k=0}^{\infty} Q_{k}(K, \cdot) \Rightarrow F(K, \cdot) \sim \sum_{k=0}^{\infty} a_{k} \vartheta_{k} Q_{k}(K, \cdot)
$$

Due to Lemma 12 , any $Q(K, \cdot), K \in \mathcal{K}$, is the limit of a sequence $\left(Q\left(K_{i}, \cdot\right)\right)$ in $\mathcal{C}^{\infty}$, and thus the last implication holds for all $K \in \mathcal{K}$. This shows (5).

It remains to show that $\left(\left|a_{k}\right|\right)$ is increasing at most polynomially in $k$. The mapping $G: \mathcal{C}^{\infty} \rightarrow \mathbb{R}$ with $f \mapsto\|T f\|_{2}$ is a spherical distribution (generalized function) of order at most $m$. As $S^{n-1}$ is compact, there is a continuous function $g$ and a differential operator $D$ such that

$$
G(f)=\int_{S^{n-1}}(D f)(v) g(v) d v
$$

holds for all $f \in \mathcal{C}^{\infty}$. For $f(u):=P_{k}^{n}(\langle u, \cdot\rangle)$ we have

$$
\begin{aligned}
\left|G P_{k}^{n}(\langle u, \cdot\rangle)\right| & =\left\|T P_{k}^{n}(\langle u, \cdot\rangle)\right\|=\left\|a_{k} \vartheta_{k} P_{k}^{n}(\langle u, \cdot\rangle)\right\| \\
& =\left|a_{k}\right| \varpi_{n}\left(\operatorname{dim} \mathcal{H}_{k}^{n}\right)^{-1} \geq c_{29}^{-1} k^{2-n}\left|a_{k}\right|
\end{aligned}
$$

for some constant $c_{29}=c_{29}(n)>0$, where we used [21, Proposition 3.3.6 and (3.1.12)]. Hence (40) yields

$$
\left|a_{k}\right| \leq c_{29} k^{n-2}\left|G P_{k}^{n}(\langle u, \cdot\rangle)\right| \leq c_{29} \varpi_{n}^{1 / 2}\|g\| k^{n-2}\left\|D P_{k}^{n}(\langle u, \cdot\rangle)\right\| .
$$

As

$$
\frac{d}{d t} P_{k}^{n}(t)=\frac{k(k+n-2)}{n-1} P_{k-1}^{n+2}(t), \quad k \geq 1,
$$

(see [21, Lemma 3.3.9]) and $\left|P_{k}^{n}(t)\right| \leq 1$ on $[-1,1]$, we conclude that $\left(\left|a_{k}\right|\right)$ is increasing at most polynomially in $k$. 
3.2. Proofs for Sections 1.1 and 1.2. To show Theorem 2 we start with an estimate depending on Hölder norms of functions. It is an easy consequence of Hölder's inequality, merely more than a reformulation of [21, Lemma 3.4.13], but we give a proof to make the main ingredients apparent.

Proposition 13. Let $f$ and $g$ be two square integrable functions on $S^{n-1}$ with

$$
f \sim \sum_{k=0}^{\infty} f_{k}, \quad g \sim \sum_{k=0}^{\infty} a_{k} f_{k}
$$

and assume that $f_{k}=0$ whenever $a_{k}=0$. Assume further that the sequence $\left(a_{k}\right)$ satisfies (7).

Then, for any $\eta \geq 0$, we have

$$
\|f\| \leq c_{30}\|f\|_{H^{\eta}}^{\beta /(\beta+\eta)}\|g\|^{\eta /(\beta+\eta)}
$$

with the constant $c_{30}=b^{\eta /(\beta+\eta)}$.

Proof. Let $p, q>1$ be a pair of conjugate exponents $(1 / p+1 / q=1)$. For a variable $0 \leq \alpha \leq \beta$ to be determined later we get from Hölder's inequality

$$
\begin{aligned}
\|f\|^{2} & =\sum_{k=0}^{\infty}\left\|f_{k}\right\|^{2}=\sum_{k=0}^{\infty}\left((1 \vee k)^{2 \alpha}\left\|f_{k}\right\|^{2 / p}\right)\left((1 \vee k)^{-2 \alpha}\left\|f_{k}\right\|^{2 / q}\right) \\
& \leq\left(\sum_{k=0}^{\infty}(1 \vee k)^{2 \alpha p}\left\|f_{k}\right\|^{2}\right)^{1 / p}\left(\sum_{k=0}^{\infty}(1 \vee k)^{-2 \alpha q}\left\|f_{k}\right\|^{2}\right)^{1 / q}
\end{aligned}
$$

The first expression on the right hand side is $\|f\|_{H^{\alpha p}}^{2 / p}$. The second expression is not larger than $b^{2 / q}\|g\|^{2 / q}$, when $\alpha q=\beta$. The choice $q=1+\beta / \eta$ (and hence $p=1+\eta / \beta$, $\alpha=\eta \beta /(\eta+\beta))$ shows the claim.

Proof of Theorem 2. Let the assumptions of the theorem be satisfied. Proposition 13 with $f=h_{K}^{\theta}-h_{K^{\prime}}^{\theta}$ and $g=F(K, \cdot)-F\left(K^{\prime}, \cdot\right)$ gives

$$
\left\|h_{K}^{\theta}-h_{K^{\prime}}^{\theta}\right\| \leq c_{30}\|f\|_{H^{\eta}}^{\beta /(\beta+\eta)}\left\|F(K, \cdot)-F\left(K^{\prime}, \cdot\right)\right\|^{\eta /(\beta+\eta)} .
$$

For $0<\eta<3 / 2$, Proposition 7 implies

$$
\left\|h_{K}^{\theta}-h_{K^{\prime}}^{\theta}\right\| \leq c_{31}\left\|F(K, \cdot)-F\left(K^{\prime}, \cdot\right)\right\|^{\eta /(\beta+\eta)}
$$

with a constant $c_{31}=c_{31}(n, \theta, b, \beta, \eta, r, R)$. In view of (24), this shows (8). That the assumptions on $K$ and $K^{\prime}$ can be weakened in the cases $\theta \geq 2$ and $\theta \in \mathbb{N}$, follows from the corresponding statements in Proposition 7.

Proof of Theorem 3. The arguments are essentially the same as those in the proof of Theorem 2. Proposition 13 with $f=\rho_{K}^{\theta}-\rho_{K^{\prime}}^{\theta}$ and $g=F(K, \cdot)-F\left(K^{\prime}, \cdot\right)$ in combination with Corollary 8 and (25) give the assertion.

3.3. Proofs for Section 1.3. We first prove an intermediate result which refines [25, Theorem 5.1]. Let $\|\mu\|_{T V}$ be the total variation norm of a signed measure $\mu$. For a Lipschitz function $f$ set

$$
\|f\|_{L}:=\sup _{u \neq v} \frac{|f(u)-f(v)|}{|u-v|}
$$


Theorem 14. Let $n \geq 2$, and let $\mu$ be a finite signed measure and $g \in L^{2}$ such that

$$
\mu \sim \sum_{k=0}^{\infty} \mu_{k}, \quad g \sim \sum_{k=0}^{\infty} a_{k} \mu_{k}
$$

where $\mu_{k}=0$ whenever $a_{k}=0$. Assume that the sequence $\left(a_{k}\right)$ satisfies (7).

If $f \in \mathcal{C}^{\infty}$ and $\beta>0$ then

$$
\left|\int_{S^{n-1}} f d \mu\right| \leq b\|f\|_{H^{\beta}}\|g\| \text {. }
$$

If $f \in H^{\eta}$ is a Lipschitz function, $\beta>\eta$, and $\left.0<\alpha<1 /(1+\beta-\eta)\right)$, then

$$
\left|\int_{S^{n-1}} f d \mu\right| \leq c_{32}\left(\|f\|_{L}+\|f\|_{H^{\eta}}\right)\|\mu\|_{T V}^{1-\alpha}\|g\|^{\alpha}
$$

where $c_{32}=c_{32}(n, b, \beta, \alpha)$ does not depend on $f$ or $\mu$.

Proof. For this proof only, we use the notation $\|\mu\|_{H^{-\beta}}$. Due to (7), we have

$$
\|\mu\|_{H^{-\beta}}^{2}:=\sum_{k=0}^{\infty}(1 \vee k)^{-2 \beta}\left\|\mu_{k}\right\|^{2} \leq b^{2} \sum_{k=0}^{\infty}\left\|a_{k} \mu_{k}\right\|^{2}=b^{2}\|g\|^{2} .
$$

For $f \in \mathcal{C}^{\infty}$ this gives the assertion (41), as

$$
\left|\int_{S^{n-1}} f d \mu\right|=\mid \sum_{k=0}^{\infty}\left\langle\left\langle f_{k}, \mu_{k}\right\rangle|| \leq\left|\sum_{k=0}^{\infty}(1 \vee k)^{\beta}\left\|f_{k}\right\|(1 \vee k)^{-\beta}\left\|\mu_{k}\right\|\right| \leq\|f\|_{H^{\beta}}\|\mu\|_{H^{-\beta}}\right.
$$

where (27) and Hölder's inequality were used.

To show the second statement let $f \in H^{\eta}$ and $\beta>\eta$. We consider two cases. If $\|g\| \geq\|\mu\|_{T V}$, the claim follows trivially from

$$
\left|\int_{S^{n-1}} f d \mu\right| \leq\|f\|_{\infty}\|\mu\|_{T V} \leq\|f\|_{\infty}\|\mu\|_{T V}^{1-\alpha}\|g\|^{\alpha},
$$

and the fact that

$$
\|f\|_{\infty} \leq 2\|f\|_{L}+\|f\|_{H^{\eta}}
$$

To treat the case $\|g\| \leq\|\mu\|_{T V}$, We refine the arguments in HUG AND SchneIDER [25], improving their estimate $(60)$. Let $\mu^{(\tau)}$ be the Poisson transform of $\mu$ with parameter $0<\tau<1$. Instead of inequality (59) in [25], we use

$$
\left|\int f(u) \mu^{(\tau)}(u) d u\right|=\left|\left\langle f f, \mu^{(\tau)}\right\rangle\right| \leq\|f\|_{H^{\eta}}\left\|\mu^{(\tau)}\right\|_{H^{-\eta}}
$$

Like in [25], it follows from calculating the maximal value of $x \mapsto x^{\beta-\eta} \tau^{x}$ that

$$
k^{\beta-\eta} \tau^{k}(1-\tau)^{\beta-\eta} \leq\left(\frac{\beta-\eta}{e}\right)^{\beta-\eta}
$$

and thus (7) implies

$$
\left\|\mu^{(\tau)}\right\|_{H^{-\eta}}=\left(\sum_{k=0}^{\infty}(1 \vee k)^{-2 \eta} \tau^{2 k}\left\|\mu_{k}\right\|^{2}\right)^{1 / 2} \leq c_{33}(1-\tau)^{-(\beta-\eta)}\|g\|,
$$

which replaces [25, equation (60)]. The rest of the proof carries over almost literally with $\beta$ and $\|f\|_{B L}$ replaced by $\beta-\eta$ and $\|f\|_{L}+\|f\|_{H^{\eta}}$, respectively. The assumption $\|g\| \leq\|\mu\|_{T V}$ is also used in the line of arguments. 
Proof of Theorem 4. Let the assumptions of the theorem be satisfied and consider the case $\beta<3 / 2$. Let $M \subset R B^{n}$ be a convex body with $h_{M} \in \mathcal{C}^{\infty}$, and put $f=h_{M}$, $\mu=S_{n-1}(K, \cdot)-S_{n-1}\left(K^{\prime}, \cdot\right)$, and $g=F(K, \cdot)-F\left(K^{\prime}, \cdot\right)$ in Theorem 14. Then, (41) and Proposition $7(\mathrm{c})$ yield

$$
\left|\int_{S^{n-1}} h_{M}(u) S_{n-1}(K, d u)-\int_{S^{n-1}} h_{M}(u) S_{n-1}\left(K^{\prime}, d u\right)\right| \leq c_{34}\left\|F(K, \cdot)-F\left(K^{\prime}, \cdot\right)\right\|
$$

with $c_{34}=c_{34}(n, b, \beta, R)$. By a usual approximation argument, (42) also holds when the assumption $h_{M} \in \mathcal{C}^{\infty}$ is dropped. Like in [25, proof of Theorem 5.2], an application of DisKANT's stability result [11] implies that there is an $x \in \mathbb{R}^{n}$ such that

$$
\delta\left(K+x, K^{\prime}\right) \leq c_{35}\left\|F(K, \cdot)-F\left(K^{\prime}, \cdot\right)\right\|^{1 / n},
$$

with $c_{35}=c_{35}(n, b, \beta, r, R)$. By assumption, $K, K^{\prime} \in \mathcal{K}_{\mathbf{a}, S_{n-1}}$, and thus their Steiner points satisfy $s(K)=s\left(K^{\prime}\right)=o$. Hence, (43) implies

$$
|x|=\left|s(K+x)-s\left(K^{\prime}\right)\right| \leq \delta\left(K+x, K^{\prime}\right) \leq c_{35}\left\|F(K, \cdot)-F\left(K^{\prime}, \cdot\right)\right\|^{1 / n} .
$$

Another application of (43) finally gives

$$
\delta\left(K, K^{\prime}\right)=\left\|h_{K}+\langle x, \cdot\rangle-h_{K^{\prime}}\right\|_{\infty} \leq \delta\left(K, K^{\prime}\right)+|x| \leq 2 c_{35}\left\|F(K, \cdot)-F\left(K^{\prime}, \cdot\right)\right\|^{1 / n} .
$$

This is the desired result for $\beta<3 / 2$. The case $\beta \geq 3 / 2$ is treated similarly.

Proof of Theorem 5. Fix a convex body $K$ and consider the spherical harmonic expansion $\sum_{k=0}^{\infty} h_{k}$ of $h_{K}$. Recall that for any $k=0,1,2, \ldots$ and any $f \in \mathcal{H}_{k}^{n}$, we have $\square f=\lambda_{k} f$, where

$$
\lambda_{k}=-\frac{(k-1)(k-1+n)}{n-1} .
$$

Due to $(30), S_{1}(K, \cdot) \sim \sum_{k=0}^{\infty} \lambda_{k} h_{k}$ and thus $F(K, \cdot) \sim \sum_{k=0}^{\infty} a_{k} \lambda_{k} \vartheta_{k} h_{k}$. This shows that $F(K, \cdot)$ can be seen as a multiplier-rotation operator depending on the support function. Its multipliers satisfy

$$
\left|a_{k} \lambda_{k}\right|^{-1} \leq 2(n-1) b(1 \vee k)^{\beta-2}, \quad \text { whenever } a_{k} \neq 0 \text { and } k \neq 1 .
$$

If $\beta \leq 2$, this implies that the finite members of the sequence $\left(\left|a_{k} \lambda_{k}\right|^{-1}\right)$ are uniformly bounded by $2(n-1) b$ and the claim follows from Parseval's identity. For $\beta>2$ Theorem 2 with $\theta=1$ and with $\beta$ replaced by $\beta-2$ implies the assumption. Note that in both cases, we have

$$
\mathcal{K}_{\mathbf{a}, S_{1}}=\left\{K \in \mathcal{K}_{\mathbf{a}, h}: s(K)=o\right\},
$$

which follows from the fact that among all multipliers $\lambda_{k}$, only the one for $k=1$ vanishes.

Proof of Theorem 6 . We treat only the case $\beta \geq 3 / 2$; the other case can be shown essentially the same way. The constants involved here depend only on $n, b, \beta, r, R$ and $\gamma$. The proof follows the proof of [25, Theorem 5.3] with two modifications. Firstly, the exponent $\alpha \in(0,2 /(n+4))$ now ranges in $(0,1 /(\beta-1 / 2))$, as Theorem 14 is applied. Secondly, we adjust the last step of the proof in [32, p. 403]. For the reader's convenience, we repeat this step, which shows that

$$
\delta\left(K, \lambda K^{\prime}\right) \leq c_{36} \bar{w}(K) \varepsilon^{q}
$$

(with $\lambda=\bar{w}(K) / \bar{w}\left(K^{\prime}\right), q=1 /\left((n+1) 2^{j-1}\right)$, and $\left.\varepsilon=\left\|F(K, \cdot)-F\left(K^{\prime}, \cdot\right)\right\|^{\alpha}\right)$ implies

$$
\delta\left(K, K^{\prime}\right) \leq c_{37} \varepsilon^{q}
$$


for sufficiently small $\varepsilon$ (where $c_{36}$ and $c_{37}$ are some constants and $K^{\prime}$ is already translated suitably). The equations

$$
V_{j}(K)=\frac{1}{\varpi_{n-j}}\left(\begin{array}{c}
n-1 \\
j
\end{array}\right) S_{j}\left(K, S^{n-1}\right)=\frac{\varpi_{n}}{\varpi_{n-j}}\left(\begin{array}{c}
n-1 \\
j
\end{array}\right)\left(S_{j}(K, \cdot)\right)_{0}
$$

show that the $j$-th intrinsic volume is up to a multiplicative constant the first term in the spherical harmonic expansion of $S_{j}(K, \cdot)$. Hence

$$
\left|a_{0}\right|\left|V_{j}(K)-V_{j}\left(K^{\prime}\right)\right| \leq c_{38}\left\|F(K, \cdot)-F\left(K^{\prime}, \cdot\right)\right\| .
$$

If $a_{0}$ would vanish, then any $M \in \mathcal{K}_{\mathbf{a}, S_{j}}$ would satisfy $V_{j}(M)=0$ and $\operatorname{dim} M \geq j$, which is impossible. Therefore $a_{0} \neq 0$ and we may conclude

$$
V_{j}(K) \leq c_{39} \varepsilon^{1 / \alpha}+V_{j}\left(K^{\prime}\right) .
$$

Due to (44), we have

$$
\lambda K^{\prime} \subset K+\left(c_{36} \bar{w}(K) \varepsilon^{q}\right) B^{n} \subset\left(1+c_{40} \varepsilon^{q}\right) K,
$$

and the fact that intrinsic volumes are monotone with respect to set inclusion implies

$$
\lambda^{j} V_{j}\left(K^{\prime}\right) \leq\left(1+c_{40} \varepsilon^{q}\right)^{j} V_{j}(K) \leq\left(1+c_{40} \varepsilon^{q}\right)^{j}\left(c_{39} \varepsilon^{1 / \alpha}+V_{j}\left(K^{\prime}\right)\right) .
$$

This gives $\lambda \leq 1+c_{41} \varepsilon^{q}$ and therefore

$$
K \subset \lambda K^{\prime}+\left(c_{36} \bar{w}(K) \varepsilon^{q}\right) B^{n} \subset K^{\prime}+c_{42} \varepsilon^{q} B^{n} .
$$

Similarly we obtain $K^{\prime} \subset K+c_{43} \varepsilon^{q} B^{n}$ and thus (45).

\section{REFERENCES}

1. A.D. Aleksandrov, On the theory of mixed volumes. New inequalities between mixed volumes and their applications.(Russian), Mat. Sb. 44 (1937), 1205-1238.

2. Yu.E. Anikonov and V.N. Stepanov, Uniqueness and stability of the solution of a problem of geometry in the large, Math. USSR-Sb. 44 (1983), 483-490.

3. _ The geometry of convex surfaces and inverse problems of scattering theory, Sib. Math. J. 35 (1994), 845-862.

4. C. Berg, Corps convexes et potentiels sphériques, Danske Vid. Selskab. Mat.-fys. Medd. 37 (1969), no. $6,64 \mathrm{p}$.

5. J. Bourgain and J. Lindenstrauss, Projection bodies, Geometric Aspects of Functional Analysis (1986/7), Lecture Notes in Math., no. 1317, Springer, Berlin, 1988, pp. 250-270.

6. S. Campi, On the reconstruction of a function on the sphere by its integrals over great circles, Boll. Unione Mat. Ital., V. Ser., C, Anal. Funz. Appl. 18 (1981), 195-215.

7. __ Reconstructing a convex surface from certain measurements of its projections, Boll. Unione Mat. Ital., VI., B 5 (1986), 945-959.

8. _ Recovering a centered convex body from the areas of its shadows: a stability estimate, Ann. Mat. Pura Appl., IV. 151 (1988), 289-302.

9. __ Stability estimates for star bodies in terms of their intersection bodies, Mathematika 45 (1998), 287-303.

10. Convex intersection bodies in three and four dimensions, Mathematika 46 (1999), 15-27.

11. V.I. Diskant, Bounds for the discrepancy between convex bodies in terms of the isoperimetric difference, Siberian Math. J. 13 (1972), 529-532.

12. W.J. Firey, p-means of convex bodies, Math. Scand. 10 (1962), 17-24.

13. P. Goodey and W. Weil, The determination of convex bodies from the mean of random sections, Math. Proc. Camb. Phil. Soc. 112 (1992), 419-430.

14. __ Average section functions for star-shaped sets, Adv. Appl. Math. 36 (2006), 70-84.

15. D__ Determination of convex bodies by directed projection functions, To appear (2006).

16. __ Directed projection functions of convex bodies, Monatsh. Math. 149 (2006), 43-64, Erratum Monatsh. Math. 149 (2006), 65.

17. P.R. Goodey, Minkowski sums of projections of convex bodies, Mathematika 45 (1998), 253-268. 
18. P.R. Goodey and H. Groemer, Stability results for first order projection bodies, Proc. Am. Math. Soc. 109 (1990), 1103-1114.

19. P.R. Goodey, M. Kiderlen, and W. Weil, Integral transforms in geometric tomography, In preparation (2007).

20. H. Groemer, Stability results for convex bodies and related spherical integral transformations, Adv. Math. 109 (1994), 45-74.

21. __ Geometric Applications of Fourier Series and Spherical Harmonics, Cambridge University Press, New York, 1996.

22. __ On the girth of convex bodies, Arch. Math. 69 (1997), 75-81.

23. 117-124.

24. J. Hoffmann-Jørgensen, Probability with a View towards Statistics, vol. 1, Chapman \& Hall, New York, 1994.

25. D. Hug and R. Schneider, Stability results involving surface area measures of convex bodies, Rend. Circ. Mat. Palermo (2) Suppl. 70 (2002), 21-51.

26. M. Kiderlen, Determination of a convex body from Minkowski sums of its projections, J. London Math. Soc. (2) $\mathbf{7 0}$ (2004), 529-544.

27. _ Blaschke- and Minkowski-endomorphisms of convex bodies, Trans. Amer. Math. Soc. 358 (2005), 5539-5564.

28. __ Mean shape estimation for particle processes from vertical sections: uniqueness and stability, In preparation (2007).

29. B. Rubin, Generalized Minkowski-Funk transforms and small denominators on the sphere, Fract. Calc. Appl. Anal. 3 (2000), 177-203.

30. R. Schneider, Über eine Integralgleichung in der Theorie der konvexen Körper, Math. Nachr. 44 (1970), 55-75.

31. Equivariant endomorphisms of the space of convex bodies, Trans. Amer. Math. Soc. 194 (1974), 53-78.

32. _ Convex Bodies: the Brunn-Minkowski Theory, Cambridge University Press, Cambridge, 1993.

33. __ On the mean normal measures of a particle process, Adv. Appl. Prob. 33 (2001), 25-38.

34. _ Stable determination of convex bodies from projections, Monatshefte Math. (2006), To appear.

35. F.E. Schuster, Convolutions and multiplier transformations of convex bodies, To appear (2006).

36. R.T. Seeley, Spherical harmonics, Am. Math. Monthly 73 (1966), 115-121.

37. E. Spodarev, On the rose of intersection of stationary flat processes, Adv. Appl. Prob. 33 (2001), $584-599$.

38. K.K. Spriestersbach, Determination of a convex body from the average of projections and stability results, Trans. Amer. Math. Soc. 194 (1998), 53-78.

39. V.N. Stepanov, First-kind equations on a sphere and some problems of convex geometry, J. Inv. Ill-Posed Problems 11 (2003), 189-310.

40. W. Weil, On surface area measures of convex bodies, Geom. Dedikata 9 (1980), 299-306.

Department of Mathematical Sciences, University of Aarhus, Ny Munkegade, DK8000 Aarhus C, Denmark

E-mail address: kiderlen@imf.au.dk 